\title{
A tutorial on support vector regression*
}

\author{
ALEX J. SMOLA and BERNHARD SCHÖLKOPF \\ RSISE, Australian National University, Canberra 0200, Australia \\ Alex.Smola@anu.edu.au \\ Max-Planck-Institut für biologische Kybernetik, 72076 Tübingen, Germany \\ Bernhard.Schoelkopf@tuebingen.mpg.de
}

Received July 2002 and accepted November 2003

\begin{abstract}
In this tutorial we give an overview of the basic ideas underlying Support Vector (SV) machines for function estimation. Furthermore, we include a summary of currently used algorithms for training SV machines, covering both the quadratic (or convex) programming part and advanced methods for dealing with large datasets. Finally, we mention some modifications and extensions that have been applied to the standard SV algorithm, and discuss the aspect of regularization from a SV perspective.
\end{abstract}

Keywords: machine learning, support vector machines, regression estimation

\section{Introduction}

The purpose of this paper is twofold. It should serve as a selfcontained introduction to Support Vector regression for readers new to this rapidly developing field of research. ${ }^{1}$ On the other hand, it attempts to give an overview of recent developments in the field.

To this end, we decided to organize the essay as follows. We start by giving a brief overview of the basic techniques in Sections 1, 2 and 3, plus a short summary with a number of figures and diagrams in Section 4. Section 5 reviews current algorithmic techniques used for actually implementing SV machines. This may be of most interest for practitioners. The following section covers more advanced topics such as extensions of the basic SV algorithm, connections between SV machines and regularization and briefly mentions methods for carrying out model selection. We conclude with a discussion of open questions and problems and current directions of SV research. Most of the results presented in this review paper already have been published elsewhere, but the comprehensive presentations and some details are new.

\subsection{Historic background}

The SV algorithm is a nonlinear generalization of the Generalized Portrait algorithm developed in Russia in the sixties ${ }^{2}$

\footnotetext{
*An extended version of this paper is available as NeuroCOLT Technical Report TR-98-030.
}

(Vapnik and Lerner 1963, Vapnik and Chervonenkis 1964). As such, it is firmly grounded in the framework of statistical learning theory, or VC theory, which has been developed over the last three decades by Vapnik and Chervonenkis (1974) and Vapnik (1982, 1995). In a nutshell, VC theory characterizes properties of learning machines which enable them to generalize well to unseen data.

In its present form, the SV machine was largely developed at AT\&T Bell Laboratories by Vapnik and co-workers (Boser, Guyon and Vapnik 1992, Guyon, Boser and Vapnik 1993, Cortes and Vapnik, 1995, Schölkopf, Burges and Vapnik 1995, 1996, Vapnik, Golowich and Smola 1997). Due to this industrial context, SV research has up to date had a sound orientation towards real-world applications. Initial work focused on OCR (optical character recognition). Within a short period of time, SV classifiers became competitive with the best available systems for both OCR and object recognition tasks (Schölkopf, Burges and Vapnik 1996, 1998a, Blanz et al. 1996, Schölkopf 1997). A comprehensive tutorial on SV classifiers has been published by Burges (1998). But also in regression and time series prediction applications, excellent performances were soon obtained (Müller et al. 1997, Drucker et al. 1997, Stitson et al. 1999, Mattera and Haykin 1999). A snapshot of the state of the art in SV learning was recently taken at the annual Neural Information Processing Systems conference (Schölkopf, Burges, and Smola 1999a). SV learning has now evolved into an active area of research. Moreover, it is in the process of entering the standard methods toolbox of machine learning (Haykin 1998, Cherkassky and Mulier 1998, Hearst et al. 1998). Schölkopf and 
Smola (2002) contains a more in-depth overview of SVM regression. Additionally, Cristianini and Shawe-Taylor (2000) and Herbrich (2002) provide further details on kernels in the context of classification.

\subsection{The basic idea}

Suppose we are given training data $\left\{\left(x_{1}, y_{1}\right), \ldots,\left(x_{\ell}, y_{\ell}\right)\right\} \subset$ $\mathcal{X} \times \mathbb{R}$, where $\mathcal{X}$ denotes the space of the input patterns (e.g. $\mathcal{X}=\mathbb{R}^{d}$ ). These might be, for instance, exchange rates for some currency measured at subsequent days together with corresponding econometric indicators. In $\varepsilon$-SV regression (Vapnik 1995), our goal is to find a function $f(x)$ that has at most $\varepsilon$ deviation from the actually obtained targets $y_{i}$ for all the training data, and at the same time is as flat as possible. In other words, we do not care about errors as long as they are less than $\varepsilon$, but will not accept any deviation larger than this. This may be important if you want to be sure not to lose more than $\varepsilon$ money when dealing with exchange rates, for instance.

For pedagogical reasons, we begin by describing the case of linear functions $f$, taking the form

$$
f(x)=\langle w, x\rangle+b \text { with } w \in \mathcal{X}, b \in \mathbb{R}
$$

where $\langle\cdot, \cdot\rangle$ denotes the dot product in $\mathcal{X}$. Flatness in the case of (1) means that one seeks a small $w$. One way to ensure this is to minimize the norm, ${ }^{3}$ i.e. $\|w\|^{2}=\langle w, w\rangle$. We can write this problem as a convex optimization problem:

$$
\begin{array}{ll}
\text { minimize } & \frac{1}{2}\|w\|^{2} \\
\text { subject to } & \left\{\begin{array}{l}
y_{i}-\left\langle w, x_{i}\right\rangle-b \leq \varepsilon \\
\left\langle w, x_{i}\right\rangle+b-y_{i} \leq \varepsilon
\end{array}\right.
\end{array}
$$

The tacit assumption in (2) was that such a function $f$ actually exists that approximates all pairs $\left(x_{i}, y_{i}\right)$ with $\varepsilon$ precision, or in other words, that the convex optimization problem is feasible. Sometimes, however, this may not be the case, or we also may want to allow for some errors. Analogously to the "soft margin" loss function (Bennett and Mangasarian 1992) which was used in SV machines by Cortes and Vapnik (1995), one can introduce slack variables $\xi_{i}, \xi_{i}^{*}$ to cope with otherwise infeasible constraints of the optimization problem (2). Hence we arrive at the formulation stated in Vapnik (1995).

$$
\begin{array}{ll}
\operatorname{minimize} & \frac{1}{2}\|w\|^{2}+C \sum_{i=1}^{\ell}\left(\xi_{i}+\xi_{i}^{*}\right) \\
\text { subject to } & \left\{\begin{array}{l}
y_{i}-\left\langle w, x_{i}\right\rangle-b \leq \varepsilon+\xi_{i} \\
\left\langle w, x_{i}\right\rangle+b-y_{i} \leq \varepsilon+\xi_{i}^{*} \\
\xi_{i}, \xi_{i}^{*}
\end{array}\right.
\end{array}
$$

The constant $C>0$ determines the trade-off between the flatness of $f$ and the amount up to which deviations larger than $\varepsilon$ are tolerated. This corresponds to dealing with a so called $\varepsilon$-insensitive loss function $|\xi|_{\varepsilon}$ described by

$$
|\xi|_{\varepsilon}:= \begin{cases}0 & \text { if }|\xi| \leq \varepsilon \\ |\xi|-\varepsilon & \text { otherwise }\end{cases}
$$

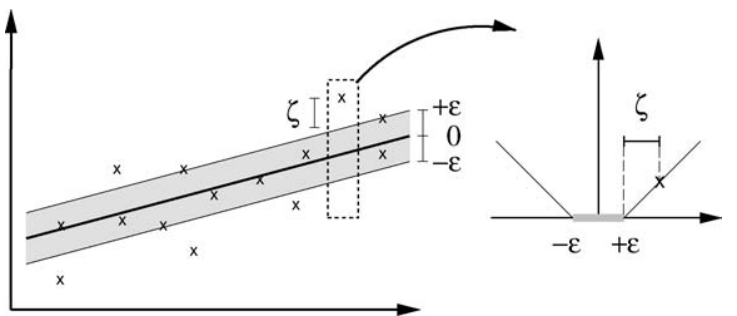

Fig. 1. The soft margin loss setting for a linear SVM (from Schölkopf and Smola, 2002)

Figure 1 depicts the situation graphically. Only the points outside the shaded region contribute to the cost insofar, as the deviations are penalized in a linear fashion. It turns out that in most cases the optimization problem (3) can be solved more easily in its dual formulation. ${ }^{4}$ Moreover, as we will see in Section 2, the dual formulation provides the key for extending SV machine to nonlinear functions. Hence we will use a standard dualization method utilizing Lagrange multipliers, as described in e.g. Fletcher (1989).

\subsection{Dual problem and quadratic programs}

The key idea is to construct a Lagrange function from the objective function (it will be called the primal objective function in the rest of this article) and the corresponding constraints, by introducing a dual set of variables. It can be shown that this function has a saddle point with respect to the primal and dual variables at the solution. For details see e.g. Mangasarian (1969), McCormick (1983), and Vanderbei (1997) and the explanations in Section 5.2. We proceed as follows:

$$
\begin{aligned}
L:= & \frac{1}{2}\|w\|^{2}+C \sum_{i=1}^{\ell}\left(\xi_{i}+\xi_{i}^{*}\right)-\sum_{i=1}^{\ell}\left(\eta_{i} \xi_{i}+\eta_{i}^{*} \xi_{i}^{*}\right) \\
& -\sum_{i=1}^{\ell} \alpha_{i}\left(\varepsilon+\xi_{i}-y_{i}+\left\langle w, x_{i}\right\rangle+b\right) \\
& -\sum_{i=1}^{\ell} \alpha_{i}^{*}\left(\varepsilon+\xi_{i}^{*}+y_{i}-\left\langle w, x_{i}\right\rangle-b\right)
\end{aligned}
$$

Here $L$ is the Lagrangian and $\eta_{i}, \eta_{i}^{*}, \alpha_{i}, \alpha_{i}^{*}$ are Lagrange multipliers. Hence the dual variables in (5) have to satisfy positivity constraints, i.e.

$$
\alpha_{i}^{(*)}, \eta_{i}^{(*)} \geq 0
$$

Note that by $\alpha_{i}^{(*)}$, we refer to $\alpha_{i}$ and $\alpha_{i}^{*}$.

It follows from the saddle point condition that the partial derivatives of $L$ with respect to the primal variables $\left(w, b, \xi_{i}, \xi_{i}^{*}\right)$ have to vanish for optimality.

$$
\begin{aligned}
\partial_{b} L & =\sum_{i=1}^{\ell}\left(\alpha_{i}^{*}-\alpha_{i}\right)=0 \\
\partial_{w} L & =w-\sum_{i=1}^{\ell}\left(\alpha_{i}-\alpha_{i}^{*}\right) x_{i}=0 \\
\partial_{\xi_{i}(*)} L & =C-\alpha_{i}^{(*)}-\eta_{i}^{(*)}=0
\end{aligned}
$$


Substituting (7), (8), and (9) into (5) yields the dual optimization problem.

$$
\begin{array}{ll}
\operatorname{maximize} & \left\{\begin{array}{l}
-\frac{1}{2} \sum_{i, j=1}^{\ell}\left(\alpha_{i}-\alpha_{i}^{*}\right)\left(\alpha_{j}-\alpha_{j}^{*}\right)\left\langle x_{i}, x_{j}\right\rangle \\
-\varepsilon \sum_{i=1}^{\ell}\left(\alpha_{i}+\alpha_{i}^{*}\right)+\sum_{i=1}^{\ell} y_{i}\left(\alpha_{i}-\alpha_{i}^{*}\right)
\end{array}\right. \\
\text { subject to } & \sum_{i=1}^{\ell}\left(\alpha_{i}-\alpha_{i}^{*}\right)=0 \quad \text { and } \alpha_{i}, \alpha_{i}^{*} \in[0, C]
\end{array}
$$

In deriving (10) we already eliminated the dual variables $\eta_{i}, \eta_{i}^{*}$ through condition (9) which can be reformulated as $\eta_{i}^{(*)}=C-$ $\alpha_{i}^{(*)}$. Equation (8) can be rewritten as follows

$w=\sum_{i=1}^{\ell}\left(\alpha_{i}-\alpha_{i}^{*}\right) x_{i}$, thus $f(x)=\sum_{i=1}^{\ell}\left(\alpha_{i}-\alpha_{i}^{*}\right)\left\langle x_{i}, x\right\rangle+b$.

This is the so-called Support Vector expansion, i.e. $w$ can be completely described as a linear combination of the training patterns $x_{i}$. In a sense, the complexity of a function's representation by SVs is independent of the dimensionality of the input space $\mathcal{X}$, and depends only on the number of SVs.

Moreover, note that the complete algorithm can be described in terms of dot products between the data. Even when evaluating $f(x)$ we need not compute $w$ explicitly. These observations will come in handy for the formulation of a nonlinear extension.

\subsection{Computing $b$}

So far we neglected the issue of computing $b$. The latter can be done by exploiting the so called Karush-Kuhn-Tucker (KKT) conditions (Karush 1939, Kuhn and Tucker 1951). These state that at the point of the solution the product between dual variables and constraints has to vanish.

$$
\begin{array}{r}
\alpha_{i}\left(\varepsilon+\xi_{i}-y_{i}+\left\langle w, x_{i}\right\rangle+b\right)=0 \\
\alpha_{i}^{*}\left(\varepsilon+\xi_{i}^{*}+y_{i}-\left\langle w, x_{i}\right\rangle-b\right)=0
\end{array}
$$

and

$$
\begin{gathered}
\left(C-\alpha_{i}\right) \xi_{i}=0 \\
\left(C-\alpha_{i}^{*}\right) \xi_{i}^{*}=0 .
\end{gathered}
$$

This allows us to make several useful conclusions. Firstly only samples $\left(x_{i}, y_{i}\right)$ with corresponding $\alpha_{i}^{(*)}=C$ lie outside the $\varepsilon$ insensitive tube. Secondly $\alpha_{i} \alpha_{i}^{*}=0$, i.e. there can never be a set of dual variables $\alpha_{i}, \alpha_{i}^{*}$ which are both simultaneously nonzero. This allows us to conclude that

$$
\begin{array}{lll}
\varepsilon-y_{i}+\left\langle w, x_{i}\right\rangle+b \geq 0 & \text { and } \quad \xi_{i}=0 & \text { if } \alpha_{i}<C \\
\varepsilon-y_{i}+\left\langle w, x_{i}\right\rangle+b \leq 0 & & \text { if } \alpha_{i}>0
\end{array}
$$

In conjunction with an analogous analysis on $\alpha_{i}^{*}$ we have

$$
\begin{aligned}
& \max \left\{-\varepsilon+y_{i}-\left\langle w, x_{i}\right\rangle \mid \alpha_{i}<C \text { or } \alpha_{i}^{*}>0\right\} \leq b \leq \\
& \min \left\{-\varepsilon+y_{i}-\left\langle w, x_{i}\right\rangle \mid \alpha_{i}>0 \text { or } \alpha_{i}^{*}<C\right\}
\end{aligned}
$$

If some $\alpha_{i}^{(*)} \in(0, C)$ the inequalities become equalities. See also Keerthi et al. (2001) for further means of choosing $b$.

Another way of computing $b$ will be discussed in the context of interior point optimization (cf. Section 5). There $b$ turns out to be a by-product of the optimization process. Further considerations shall be deferred to the corresponding section. See also Keerthi et al. (1999) for further methods to compute the constant offset.

A final note has to be made regarding the sparsity of the SV expansion. From (12) it follows that only for $\left|f\left(x_{i}\right)-y_{i}\right| \geq \varepsilon$ the Lagrange multipliers may be nonzero, or in other words, for all samples inside the $\varepsilon$-tube (i.e. the shaded region in Fig. 1) the $\alpha_{i}, \alpha_{i}^{*}$ vanish: for $\left|f\left(x_{i}\right)-y_{i}\right|<\varepsilon$ the second factor in (12) is nonzero, hence $\alpha_{i}, \alpha_{i}^{*}$ has to be zero such that the KKT conditions are satisfied. Therefore we have a sparse expansion of $w$ in terms of $x_{i}$ (i.e. we do not need all $x_{i}$ to describe $w$ ). The examples that come with nonvanishing coefficients are called Support Vectors.

\section{Kernels}

\subsection{Nonlinearity by preprocessing}

The next step is to make the SV algorithm nonlinear. This, for instance, could be achieved by simply preprocessing the training patterns $x_{i}$ by a map $\Phi: \mathcal{X} \rightarrow \mathcal{F}$ into some feature space $\mathcal{F}$, as described in Aizerman, Braverman and Rozonoér (1964) and Nilsson (1965) and then applying the standard SV regression algorithm. Let us have a brief look at an example given in Vapnik (1995).

Example 1 (Quadratic features in $\mathbb{R}^{2}$ ). Consider the map $\Phi$ : $\mathbb{R}^{2} \rightarrow \mathbb{R}^{3}$ with $\Phi\left(x_{1}, x_{2}\right)=\left(x_{1}^{2}, \sqrt{2} x_{1} x_{2}, x_{2}^{2}\right)$. It is understood that the subscripts in this case refer to the components of $x \in \mathbb{R}^{2}$. Training a linear SV machine on the preprocessed features would yield a quadratic function.

While this approach seems reasonable in the particular example above, it can easily become computationally infeasible for both polynomial features of higher order and higher dimensionality, as the number of different monomial features of degree $p$ is $\left({ }^{d+p}-1\right)$, where $d=\operatorname{dim}(\mathcal{X})$. Typical values for OCR tasks (with good performance) (Schölkopf, Burges and Vapnik 1995, Schölkopf et al. 1997, Vapnik 1995) are $p=7, d=28 \cdot 28=784$, corresponding to approximately $3.7 \cdot 10^{16}$ features.

\subsection{Implicit mapping via kernels}

Clearly this approach is not feasible and we have to find a computationally cheaper way. The key observation (Boser, Guyon 
and Vapnik 1992) is that for the feature map of example 2.1 we have

$$
\left\langle\left(x_{1}^{2}, \sqrt{2} x_{1} x_{2}, x_{2}^{2}\right),\left(x_{1}^{\prime 2}, \sqrt{2} x_{1}^{\prime} x_{2}^{\prime}, x_{2}^{\prime 2}\right)\right\rangle=\left\langle x, x^{\prime}\right\rangle^{2} .
$$

As noted in the previous section, the SV algorithm only depends on dot products between patterns $x_{i}$. Hence it suffices to know $k\left(x, x^{\prime}\right):=\left\langle\Phi(x), \Phi\left(x^{\prime}\right)\right\rangle$ rather than $\Phi$ explicitly which allows us to restate the $\mathrm{SV}$ optimization problem:

$$
\operatorname{maximize}\left\{\begin{array}{l}
-\frac{1}{2} \sum_{i, j=1}^{\ell}\left(\alpha_{i}-\alpha_{i}^{*}\right)\left(\alpha_{j}-\alpha_{j}^{*}\right) k\left(x_{i}, x_{j}\right) \\
-\varepsilon \sum_{i=1}^{\ell}\left(\alpha_{i}+\alpha_{i}^{*}\right)+\sum_{i=1}^{\ell} y_{i}\left(\alpha_{i}-\alpha_{i}^{*}\right)
\end{array}\right.
$$$$
\text { subject to } \sum_{i=1}^{\ell}\left(\alpha_{i}-\alpha_{i}^{*}\right)=0 \quad \text { and } \quad \alpha_{i}, \alpha_{i}^{*} \in[0, C]
$$

Likewise the expansion of $f$ (11) may be written as

$$
\begin{aligned}
w & =\sum_{i=1}^{\ell}\left(\alpha_{i}-\alpha_{i}^{*}\right) \Phi\left(x_{i}\right) \quad \text { and } \\
f(x) & =\sum_{i=1}^{\ell}\left(\alpha_{i}-\alpha_{i}^{*}\right) k\left(x_{i}, x\right)+b .
\end{aligned}
$$

The difference to the linear case is that $w$ is no longer given explicitly. Also note that in the nonlinear setting, the optimization problem corresponds to finding the flattest function in feature space, not in input space.

\subsection{Conditions for kernels}

The question that arises now is, which functions $k\left(x, x^{\prime}\right)$ correspond to a dot product in some feature space $\mathcal{F}$. The following theorem characterizes these functions (defined on $\mathcal{X}$ ).

Theorem 2 (Mercer 1909). Suppose $k \in L_{\infty}\left(\mathcal{X}^{2}\right)$ such that the integral operator $T_{k}: L_{2}(\mathcal{X}) \rightarrow L_{2}(\mathcal{X})$,

$$
T_{k} f(\cdot):=\int_{\mathcal{X}} k(\cdot, x) f(x) d \mu(x)
$$

is positive (here $\mu$ denotes a measure on $\mathcal{X}$ with $\mu(\mathcal{X})$ finite and $\operatorname{supp}(\mu)=\mathcal{X})$. Let $\psi_{j} \in L_{2}(\mathcal{X})$ be the eigenfunction of $T_{k}$ associated with the eigenvalue $\lambda_{j} \neq 0$ and normalized such that $\left\|\psi_{j}\right\|_{L_{2}}=1$ and let $\overline{\psi_{j}}$ denote its complex conjugate. Then

1. $\left(\lambda_{j}(T)\right)_{j} \in \ell_{1}$.

2. $k\left(x, x^{\prime}\right)=\sum_{j \in \mathbb{N}} \lambda_{j} \overline{\psi_{j}(x)} \psi_{j}\left(x^{\prime}\right)$ holds for almost all $\left(x, x^{\prime}\right)$, where the series converges absolutely and uniformly for almost all $\left(x, x^{\prime}\right)$.

Less formally speaking this theorem means that if

$$
\int_{\mathcal{X} \times \mathcal{X}} k\left(x, x^{\prime}\right) f(x) f\left(x^{\prime}\right) d x d x^{\prime} \geq 0 \text { for all } f \in L_{2}(\mathcal{X})
$$

holds we can write $k\left(x, x^{\prime}\right)$ as a dot product in some feature space. From this condition we can conclude some simple rules for compositions of kernels, which then also satisfy Mercer's condition (Schölkopf, Burges and Smola 1999a). In the following we will call such functions $k$ admissible SV kernels.

Corollary 3 (Positive linear combinations of kernels). Denote by $k_{1}, k_{2}$ admissible SV kernels and $c_{1}, c_{2} \geq 0$ then

$$
k\left(x, x^{\prime}\right):=c_{1} k_{1}\left(x, x^{\prime}\right)+c_{2} k_{2}\left(x, x^{\prime}\right)
$$

is an admissible kernel. This follows directly from (21) by virtue of the linearity of integrals.

More generally, one can show that the set of admissible kernels forms a convex cone, closed in the topology of pointwise convergence (Berg, Christensen and Ressel 1984).

Corollary 4 (Integrals of kernels). Let $s\left(x, x^{\prime}\right)$ be a function on $\mathcal{X} \times \mathcal{X}$ such that

$$
k\left(x, x^{\prime}\right):=\int_{\mathcal{X}} s(x, z) s\left(x^{\prime}, z\right) d z
$$

exists. Then $k$ is an admissible SV kernel.

This can be shown directly from (21) and (23) by rearranging the order of integration. We now state a necessary and sufficient condition for translation invariant kernels, i.e. $k\left(x, x^{\prime}\right):=k\left(x-x^{\prime}\right)$ as derived in Smola, Schölkopf and Müller (1998c).

Theorem 5 (Products of kernels). Denote by $k_{1}$ and $k_{2}$ admissible SV kernels then

$$
k\left(x, x^{\prime}\right):=k_{1}\left(x, x^{\prime}\right) k_{2}\left(x, x^{\prime}\right)
$$

is an admissible kernel.

This can be seen by an application of the "expansion part" of Mercer's theorem to the kernels $k_{1}$ and $k_{2}$ and observing that each term in the double sum $\sum_{i, j} \lambda_{i}^{1} \lambda_{j}^{2} \psi_{i}^{1}(x) \psi_{i}^{1}\left(x^{\prime}\right) \psi_{j}^{2}(x) \psi_{j}^{2}\left(x^{\prime}\right)$ gives rise to a positive coefficient when checking (21).

Theorem 6 (Smola, Schölkopf and Müller 1998c). A translation invariant kernel $k\left(x, x^{\prime}\right)=k\left(x-x^{\prime}\right)$ is an admissible $S V$ kernels if and only if the Fourier transform

$$
F[k](\omega)=(2 \pi)^{-\frac{d}{2}} \int_{\mathcal{X}} e^{-i\langle\omega, x\rangle} k(x) d x
$$

is nonnegative.

We will give a proof and some additional explanations to this theorem in Section 7. It follows from interpolation theory (Micchelli 1986) and the theory of regularization networks (Girosi, Jones and Poggio 1993). For kernels of the dot-product type, i.e. $k\left(x, x^{\prime}\right)=k\left(\left\langle x, x^{\prime}\right\rangle\right)$, there exist sufficient conditions for being admissible.

Theorem 7 (Burges 1999). Any kernel of dot-product type $k\left(x, x^{\prime}\right)=k\left(\left\langle x, x^{\prime}\right\rangle\right)$ has to satisfy

$$
k(\xi) \geq 0, \partial_{\xi} k(\xi) \geq 0 \text { and } \partial_{\xi} k(\xi)+\xi \partial_{\xi}^{2} k(\xi) \geq 0
$$

for any $\xi \geq 0$ in order to be an admissible SV kernel. 
Note that the conditions in Theorem 7 are only necessary but not sufficient. The rules stated above can be useful tools for practitioners both for checking whether a kernel is an admissible SV kernel and for actually constructing new kernels. The general case is given by the following theorem.

Theorem 8 (Schoenberg 1942). A kernel of dot-product type $k\left(x, x^{\prime}\right)=k\left(\left\langle x, x^{\prime}\right\rangle\right)$ defined on an infinite dimensional Hilbert space, with a power series expansion

$$
k(t)=\sum_{n=0}^{\infty} a_{n} t^{n}
$$

is admissible if and only if all $a_{n} \geq 0$.

A slightly weaker condition applies for finite dimensional spaces. For further details see Berg, Christensen and Ressel (1984) and Smola, Óvári and Williamson (2001).

\subsection{Examples}

In Schölkopf, Smola and Müller (1998b) it has been shown, by explicitly computing the mapping, that homogeneous polynomial kernels $k$ with $p \in \mathbb{N}$ and

$$
k\left(x, x^{\prime}\right)=\left\langle x, x^{\prime}\right\rangle^{p}
$$

are suitable SV kernels (cf. Poggio 1975). From this observation one can conclude immediately (Boser, Guyon and Vapnik 1992, Vapnik 1995) that kernels of the type

$$
k\left(x, x^{\prime}\right)=\left(\left\langle x, x^{\prime}\right\rangle+c\right)^{p}
$$

i.e. inhomogeneous polynomial kernels with $p \in \mathbb{N}, c \geq 0$ are admissible, too: rewrite $k$ as a sum of homogeneous kernels and apply Corollary 3. Another kernel, that might seem appealing due to its resemblance to Neural Networks is the hyperbolic tangent kernel

$$
k\left(x, x^{\prime}\right)=\tanh \left(\vartheta+\kappa\left\langle x, x^{\prime}\right\rangle\right)
$$

By applying Theorem 8 one can check that this kernel does not actually satisfy Mercer's condition (Ovari 2000). Curiously, the kernel has been successfully used in practice; cf. Scholkopf (1997) for a discussion of the reasons.

Translation invariant kernels $k\left(x, x^{\prime}\right)=k\left(x-x^{\prime}\right)$ are quite widespread. It was shown in Aizerman, Braverman and Rozonoér (1964), Micchelli (1986) and Boser, Guyon and Vapnik (1992) that

$$
k\left(x, x^{\prime}\right)=e^{-\frac{\left\|x-x^{\prime}\right\|^{2}}{2 \sigma^{2}}}
$$

is an admissible SV kernel. Moreover one can show (Smola 1996, Vapnik, Golowich and Smola 1997) that ( $\mathbf{1}_{X}$ denotes the indicator function on the set $X$ and $\otimes$ the convolution operation)

$$
k\left(x, x^{\prime}\right)=B_{2 n+1}\left(\left\|x-x^{\prime}\right\|\right) \text { with } B_{k}:=\bigotimes_{i=1}^{k} \mathbf{1}_{\left[-\frac{1}{2}, \frac{1}{2}\right]}
$$

$B$-splines of order $2 n+1$, defined by the $2 n+1$ convolution of the unit inverval, are also admissible. We shall postpone further considerations to Section 7 where the connection to regularization operators will be pointed out in more detail.

\section{Cost functions}

So far the SV algorithm for regression may seem rather strange and hardly related to other existing methods of function estimation (e.g. Huber 1981, Stone 1985, Härdle 1990, Hastie and Tibshirani 1990, Wahba 1990). However, once cast into a more standard mathematical notation, we will observe the connections to previous work. For the sake of simplicity we will, again, only consider the linear case, as extensions to the nonlinear one are straightforward by using the kernel method described in the previous chapter.

\subsection{The risk functional}

Let us for a moment go back to the case of Section 1.2. There, we had some training data $\mathbf{X}:=\left\{\left(x_{1}, y_{1}\right), \ldots,\left(x_{\ell}, y_{\ell}\right)\right\} \subset \mathcal{X} \times \mathbb{R}$. We will assume now, that this training set has been drawn iid (independent and identically distributed) from some probability distribution $P(x, y)$. Our goal will be to find a function $f$ minimizing the expected risk (cf. Vapnik 1982)

$$
R[f]=\int c(x, y, f(x)) d P(x, y)
$$

$(c(x, y, f(x))$ denotes a cost function determining how we will penalize estimation errors) based on the empirical data $\mathbf{X}$. Given that we do not know the distribution $P(x, y)$ we can only use $\mathbf{X}$ for estimating a function $f$ that minimizes $R[f]$. A possible approximation consists in replacing the integration by the empirical estimate, to get the so called empirical risk functional

$$
R_{\mathrm{emp}}[f]:=\frac{1}{\ell} \sum_{i=1}^{\ell} c\left(x_{i}, y_{i}, f\left(x_{i}\right)\right)
$$

A first attempt would be to find the empirical risk minimizer $f_{0}:=\operatorname{argmin}_{f \in H} R_{\text {emp }}[f]$ for some function class $H$. However, if $H$ is very rich, i.e. its "capacity" is very high, as for instance when dealing with few data in very high-dimensional spaces, this may not be a good idea, as it will lead to overfitting and thus bad generalization properties. Hence one should add a capacity control term, in the SV case $\|w\|^{2}$, which leads to the regularized risk functional (Tikhonov and Arsenin 1977, Morozov 1984, Vapnik 1982)

$$
R_{\mathrm{reg}}[f]:=R_{\mathrm{emp}}[f]+\frac{\lambda}{2}\|w\|^{2}
$$

where $\lambda>0$ is a so called regularization constant. Many algorithms like regularization networks (Girosi, Jones and Poggio 1993) or neural networks with weight decay networks (e.g. Bishop 1995) minimize an expression similar to (35). 


\subsection{Maximum likelihood and density models}

The standard setting in the SV case is, as already mentioned in Section 1.2, the $\varepsilon$-insensitive loss

$$
c(x, y, f(x))=|y-f(x)|_{\varepsilon} .
$$

It is straightforward to show that minimizing (35) with the particular loss function of (36) is equivalent to minimizing (3), the only difference being that $C=1 /(\lambda \ell)$.

Loss functions such like $|y-f(x)|_{\varepsilon}^{p}$ with $p>1$ may not be desirable, as the superlinear increase leads to a loss of the robustness properties of the estimator (Huber 1981): in those cases the derivative of the cost function grows without bound. For $p<1$, on the other hand, $c$ becomes nonconvex.

For the case of $c(x, y, f(x))=(y-f(x))^{2}$ we recover the least mean squares fit approach, which, unlike the standard SV loss function, leads to a matrix inversion instead of a quadratic programming problem.

The question is which cost function should be used in (35). On the one hand we will want to avoid a very complicated function $c$ as this may lead to difficult optimization problems. On the other hand one should use that particular cost function that suits the problem best. Moreover, under the assumption that the samples were generated by an underlying functional dependency plus additive noise, i.e. $y_{i}=f_{\text {true }}\left(x_{i}\right)+\xi_{i}$ with density $p(\xi)$, then the optimal cost function in a maximum likelihood sense is

$$
c(x, y, f(x))=-\log p(y-f(x)) .
$$

This can be seen as follows. The likelihood of an estimate

$$
\mathbf{X}_{f}:=\left\{\left(x_{1}, f\left(x_{1}\right)\right), \ldots,\left(x_{\ell}, f\left(x_{\ell}\right)\right)\right\}
$$

for additive noise and iid data is

$$
p\left(\mathbf{X}_{f} \mid \mathbf{X}\right)=\prod_{i=1}^{\ell} p\left(f\left(x_{i}\right) \mid\left(x_{i}, y_{i}\right)\right)=\prod_{i=1}^{\ell} p\left(y_{i}-f\left(x_{i}\right)\right) .
$$

Maximizing $P\left(\mathbf{X}_{f} \mid \mathbf{X}\right)$ is equivalent to minimizing $-\log P$ $\left(\mathbf{X}_{f} \mid \mathbf{X}\right)$. By using (37) we get

$$
-\log P\left(\mathbf{X}_{f} \mid \mathbf{X}\right)=\sum_{i=1}^{\ell} c\left(x_{i}, y_{i}, f\left(x_{i}\right)\right) \text {. }
$$

However, the cost function resulting from this reasoning might be nonconvex. In this case one would have to find a convex proxy in order to deal with the situation efficiently (i.e. to find an efficient implementation of the corresponding optimization problem).

If, on the other hand, we are given a specific cost function from a real world problem, one should try to find as close a proxy to this cost function as possible, as it is the performance wrt. this particular cost function that matters ultimately.

Table 1 contains an overview over some common density models and the corresponding loss functions as defined by (37).

The only requirement we will impose on $c(x, y, f(x))$ in the following is that for fixed $x$ and $y$ we have convexity in $f(x)$. This requirement is made, as we want to ensure the existence and uniqueness (for strict convexity) of a minimum of optimization problems (Fletcher 1989).

\subsection{Solving the equations}

For the sake of simplicity we will additionally assume $c$ to be symmetric and to have (at most) two (for symmetry) discontinuities at $\pm \varepsilon, \varepsilon \geq 0$ in the first derivative, and to be zero in the interval $[-\varepsilon, \varepsilon]$. All loss functions from Table 1 belong to this class. Hence $c$ will take on the following form.

$$
c(x, y, f(x))=\tilde{c}\left(|y-f(x)|_{\varepsilon}\right)
$$

Note the similarity to Vapnik's $\varepsilon$-insensitive loss. It is rather straightforward to extend this special choice to more general convex cost functions. For nonzero cost functions in the interval $[-\varepsilon, \varepsilon]$ use an additional pair of slack variables. Moreover we might choose different cost functions $\tilde{c}_{i}, \tilde{c}_{i}^{*}$ and different values of $\varepsilon_{i}, \varepsilon_{i}^{*}$ for each sample. At the expense of additional

\begin{tabular}{|c|c|c|}
\hline & Loss function & Density model \\
\hline$\varepsilon$-insensitive & $c(\xi)=|\xi|_{\varepsilon}$ & $p(\xi)=\frac{1}{2(1+\varepsilon)} \exp \left(-|\xi|_{\varepsilon}\right)$ \\
\hline Laplacian & $c(\xi)=|\xi|$ & $p(\xi)=\frac{1}{2} \exp (-|\xi|)$ \\
\hline Gaussian & $c(\xi)=\frac{1}{2} \xi^{2}$ & $p(\xi)=\frac{1}{\sqrt{2 \pi}} \exp \left(-\frac{\xi^{2}}{2}\right)$ \\
\hline Huber's robust loss & $c(\xi)= \begin{cases}\frac{1}{2 \sigma}(\xi)^{2} & \text { if }|\xi| \leq \sigma \\
|\xi|-\frac{\sigma}{2} & \text { otherwise }\end{cases}$ & $p(\xi) \propto \begin{cases}\exp \left(-\frac{\xi^{2}}{2 \sigma}\right) & \text { if }|\xi| \leq \sigma \\
\exp \left(\frac{\sigma}{2}-|\xi|\right) & \text { otherwise }\end{cases}$ \\
\hline Polynomial & $c(\xi)=\frac{1}{p}|\xi|^{p}$ & $p(\xi)=\frac{p}{2 \Gamma(1 / p)} \exp \left(-|\xi|^{p}\right)$ \\
\hline Piecewise polynomial & $c(\xi)= \begin{cases}\frac{1}{p \sigma^{p-1}}(\xi)^{p} & \text { if }|\xi| \leq \sigma \\
|\xi|-\sigma \frac{p-1}{p} & \text { otherwise }\end{cases}$ & $p(\xi) \propto \begin{cases}\exp \left(-\frac{\xi^{p}}{p \sigma^{p-1}}\right) & \text { if }|\xi| \leq \sigma \\
\exp \left(\sigma \frac{p-1}{p}-|\xi|\right) & \text { otherwise }\end{cases}$ \\
\hline
\end{tabular}
Lagrange multipliers in the dual formulation additional discontinuities also can be taken care of. Analogously to (3) we arrive at a convex minimization problem (Smola and Schölkopf 1998a). To simplify notation we will stick to the one of (3) and use $C$

Table 1. Common loss functions and corresponding density models 
instead of normalizing by $\lambda$ and $\ell$.

$$
\begin{array}{ll}
\operatorname{minimize} & \frac{1}{2}\|w\|^{2}+C \sum_{i=1}^{\ell}\left(\tilde{c}\left(\xi_{i}\right)+\tilde{c}\left(\xi_{i}^{*}\right)\right) \\
\text { subject to } & \begin{cases}y_{i}-\left\langle w, x_{i}\right\rangle-b \leq \varepsilon+\xi_{i} \\
\left\langle w, x_{i}\right\rangle+b-y_{i} & \leq \varepsilon+\xi_{i}^{*} \\
\xi_{i}, \xi_{i}^{*} & \geq 0\end{cases}
\end{array}
$$

Again, by standard Lagrange multiplier techniques, exactly in the same manner as in the $|\cdot|_{\varepsilon}$ case, one can compute the dual optimization problem (the main difference is that the slack variable terms $\tilde{c}\left(\xi_{i}^{(*)}\right)$ now have nonvanishing derivatives). We will omit the indices ${ }_{i}$ and $*$, where applicable to avoid tedious notation. This yields

$$
\begin{aligned}
& \operatorname{maximize}\left\{\begin{array}{l}
-\frac{1}{2} \sum_{i, j=1}^{\ell}\left(\alpha_{i}-\alpha_{i}^{*}\right)\left(\alpha_{j}-\alpha_{j}^{*}\right)\left\langle x_{i}, x_{j}\right\rangle \\
+\sum_{i=1}^{\ell} y_{i}\left(\alpha_{i}-\alpha_{i}^{*}\right)-\varepsilon\left(\alpha_{i}+\alpha_{i}^{*}\right) \\
+C \sum_{i=1}^{\ell} T\left(\xi_{i}\right)+T\left(\xi_{i}^{*}\right)
\end{array}\right. \\
& \text { where }\left\{\begin{array}{l}
w=\sum_{i=1}^{\ell}\left(\alpha_{i}-\alpha_{i}^{*}\right) x_{i} \\
T(\xi):=\tilde{c}(\xi)-\xi \partial_{\xi} \tilde{c}(\xi)
\end{array}\right. \\
& \text { subject to }\left\{\begin{array}{l}
\ell \\
\sum_{i=1}^{\ell}\left(\alpha_{i}-\alpha_{i}^{*}\right)=0 \\
\alpha \leq C \partial_{\xi} \tilde{c}(\xi) \\
\xi=\inf \left\{\xi \mid C \partial_{\xi} \tilde{c} \geq \alpha\right\} \\
\alpha, \xi \geq 0
\end{array}\right.
\end{aligned}
$$

\subsection{Examples}

Let us consider the examples of Table 1 . We will show explicitly for two examples how (43) can be further simplified to bring it into a form that is practically useful. In the $\varepsilon$-insensitive case, i.e. $\tilde{c}(\xi)=|\xi|$ we get

$$
T(\xi)=\xi-\xi \cdot 1=0 .
$$

Morover one can conclude from $\partial_{\xi} \tilde{c}(\xi)=1$ that

$$
\xi=\inf \{\xi \mid C \geq \alpha\}=0 \quad \text { and } \quad \alpha \in[0, C] .
$$

For the case of piecewise polynomial loss we have to distinguish two different cases: $\xi \leq \sigma$ and $\xi>\sigma$. In the first case we get

$$
T(\xi)=\frac{1}{p \sigma^{p-1}} \xi^{p}-\frac{1}{\sigma^{p-1}} \xi^{p}=-\frac{p-1}{p} \sigma^{1-p} \xi^{p}
$$

and $\xi=\inf \left\{\xi \mid C \sigma^{1-p} \xi^{p-1} \geq \alpha\right\}=\sigma C^{-\frac{1}{p-1}} \alpha^{\frac{1}{p-1}}$ and thus

$$
T(\xi)=-\frac{p-1}{p} \sigma C^{-\frac{p}{p-1}} \alpha^{\frac{p}{p-1}}
$$

Table 2. Terms of the convex optimization problem depending on the choice of the loss function

\begin{tabular}{llll}
\hline & $\varepsilon$ & $\alpha$ & $C T(\alpha)$ \\
\hline$\varepsilon$-insensitive & $\varepsilon \neq 0$ & $\alpha \in[0, C]$ & 0 \\
Laplacian & $\varepsilon=0$ & $\alpha \in[0, C]$ & 0 \\
Gaussian & $\varepsilon=0$ & $\alpha \in[0, \infty)$ & $-\frac{1}{2} C^{-1} \alpha^{2}$ \\
$\begin{array}{l}\text { Huber's } \\
\text { robust loss }\end{array}$ & $\varepsilon=0$ & $\alpha \in[0, C]$ & $-\frac{1}{2} \sigma C^{-1} \alpha^{2}$ \\
$\begin{array}{l}\text { Polynomial } \\
\text { Piecewise }\end{array}$ & $\varepsilon=0$ & $\alpha \in[0, \infty)$ & $-\frac{p-1}{p} C^{-\frac{1}{p-1}} \alpha^{\frac{p}{p-1}}$ \\
$\quad$ polynomial & $\varepsilon=0$ & $\alpha \in[0, C]$ & $-\frac{p-1}{p} \sigma C^{-\frac{1}{p-1}} \alpha^{\frac{p}{p-1}}$ \\
\hline
\end{tabular}

In the second case $(\xi \geq \sigma)$ we have

$$
T(\xi)=\xi-\sigma \frac{p-1}{p}-\xi=-\sigma \frac{p-1}{p}
$$

and $\xi=\inf \{\xi \mid C \geq \alpha\}=\sigma$, which, in turn yields $\alpha \in[0, C]$. Combining both cases we have

$$
\alpha \in[0, C] \text { and } T(\alpha)=-\frac{p-1}{p} \sigma C^{-\frac{p}{p-1}} \alpha^{\frac{p}{p-1}} .
$$

Table 2 contains a summary of the various conditions on $\alpha$ and formulas for $T(\alpha)$ (strictly speaking $T(\xi(\alpha))$ ) for different cost functions. ${ }^{5}$ Note that the maximum slope of $\tilde{c}$ determines the region of feasibility of $\alpha$, i.e. $s:=\sup _{\xi \in \mathbb{R}^{+}} \partial_{\xi} \tilde{c}(\xi)<\infty$ leads to compact intervals $[0, C s]$ for $\alpha$. This means that the influence of a single pattern is bounded, leading to robust estimators (Huber 1972). One can also observe experimentally that the performance of a SV machine depends significantly on the cost function used (Müller et al. 1997, Smola, Schölkopf and Müller 1998b)

A cautionary remark is necessary regarding the use of cost functions other than the $\varepsilon$-insensitive one. Unless $\varepsilon \neq 0$ we will lose the advantage of a sparse decomposition. This may be acceptable in the case of few data, but will render the prediction step extremely slow otherwise. Hence one will have to trade off a potential loss in prediction accuracy with faster predictions. Note, however, that also a reduced set algorithm like in Burges (1996), Burges and Schölkopf (1997) and Schölkopf et al. (1999b) or sparse decomposition techniques (Smola and Schölkopf 2000) could be applied to address this issue. In a Bayesian setting, Tipping (2000) has recently shown how an $L_{2}$ cost function can be used without sacrificing sparsity.

\section{The bigger picture}

Before delving into algorithmic details of the implementation let us briefly review the basic properties of the SV algorithm for regression as described so far. Figure 2 contains a graphical overview over the different steps in the regression stage.

The input pattern (for which a prediction is to be made) is mapped into feature space by a map $\Phi$. Then dot products are computed with the images of the training patterns under 


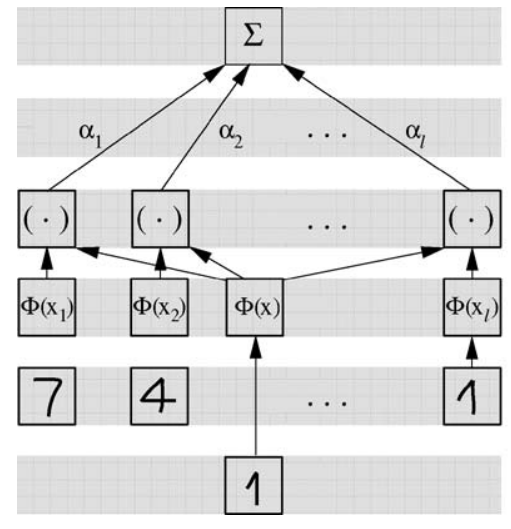

output $\Sigma \alpha_{j} k\left(\mathrm{x}, \mathrm{x}_{i}\right)+b$

weights

dot product $\left(\Phi(\mathrm{x}) \cdot \Phi\left(\mathrm{x}_{i}\right)\right)=k\left(\mathrm{x}, \mathrm{x}_{i}\right)$

mapped vectors $\Phi\left(\mathrm{x}_{i}\right), \Phi(\mathrm{x})$

support vectors $\mathrm{x}_{1} \ldots \mathrm{x}_{l}$

test vector $\mathrm{x}$

Fig. 2. Architecture of a regression machine constructed by the SV algorithm

the map $\Phi$. This corresponds to evaluating kernel functions $k\left(x_{i}, x\right)$. Finally the dot products are added up using the weights $v_{i}=\alpha_{i}-\alpha_{i}^{*}$. This, plus the constant term $b$ yields the final prediction output. The process described here is very similar to regression in a neural network, with the difference, that in the SV case the weights in the input layer are a subset of the training patterns.

Figure 3 demonstrates how the SV algorithm chooses the flattest function among those approximating the original data with a given precision. Although requiring flatness only in feature space, one can observe that the functions also are very flat in input space. This is due to the fact, that kernels can be associated with flatness properties via regular- ization operators. This will be explained in more detail in Section 7.

Finally Fig. 4 shows the relation between approximation quality and sparsity of representation in the SV case. The lower the precision required for approximating the original data, the fewer SVs are needed to encode that. The non-SVs are redundant, i.e. even without these patterns in the training set, the SV machine would have constructed exactly the same function $f$. One might think that this could be an efficient way of data compression, namely by storing only the support patterns, from which the estimate can be reconstructed completely. However, this simple analogy turns out to fail in the case of high-dimensional data, and even more drastically in the presence of noise. In Vapnik, Golowich and Smola (1997) one can see that even for moderate approximation quality, the number of SVs can be considerably high, yielding rates worse than the Nyquist rate (Nyquist 1928, Shannon 1948).

\section{Optimization algorithms}

While there has been a large number of implementations of SV algorithms in the past years, we focus on a few algorithms which will be presented in greater detail. This selection is somewhat biased, as it contains these algorithms the authors are most familiar with. However, we think that this overview contains some of the most effective ones and will be useful for practitioners who would like to actually code a SV machine by themselves. But before doing so we will briefly cover major optimization packages and strategies.
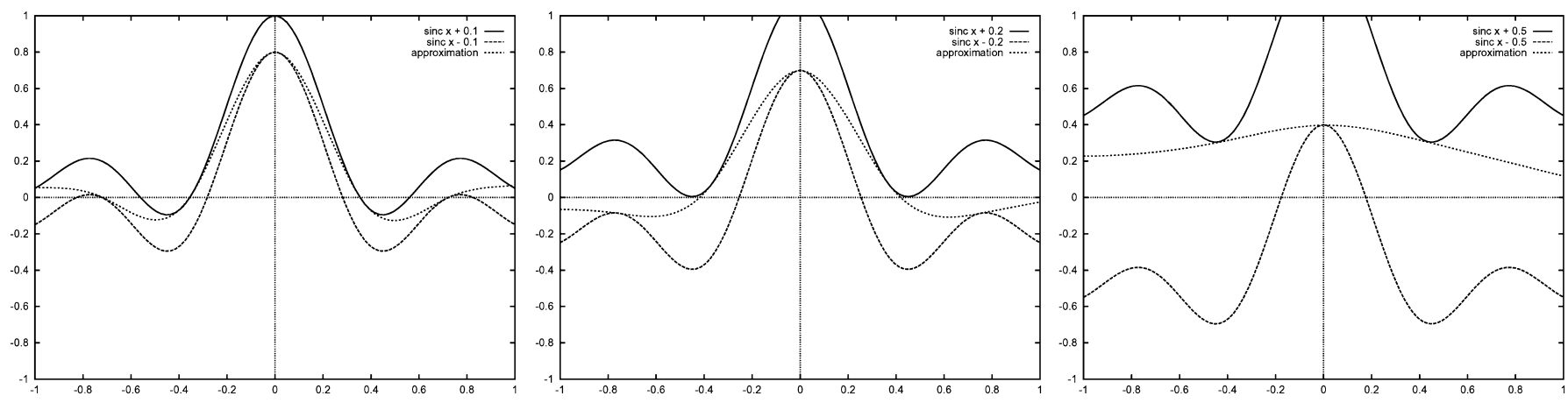

Fig. 3. Left to right: approximation of the function $\operatorname{sinc} x$ with precisions $\varepsilon=0.1,0.2$, and 0.5 . The solid top and the bottom lines indicate the size of the $\varepsilon$-tube, the dotted line in between is the regression

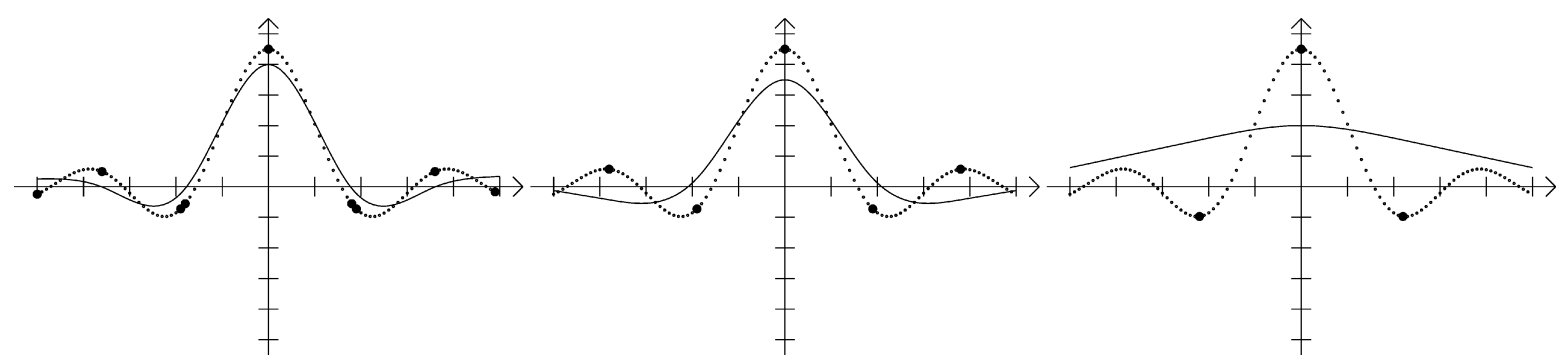

Fig. 4. Left to right: regression (solid line), datapoints (small dots) and SVs (big dots) for an approximation with $\varepsilon=0.1,0.2$, and 0.5. Note the decrease in the number of $S V S$ 


\subsection{Implementations}

Most commercially available packages for quadratic programming can also be used to train SV machines. These are usually numerically very stable general purpose codes, with special enhancements for large sparse systems. While the latter is a feature that is not needed at all in SV problems (there the dot product matrix is dense and huge) they still can be used with good success. $^{6}$

OSL: This package was written by IBM-Corporation (1992). It uses a two phase algorithm. The first step consists of solving a linear approximation of the QP problem by the simplex algorithm (Dantzig 1962). Next a related very simple QP problem is dealt with. When successive approximations are close enough together, the second subalgorithm, which permits a quadratic objective and converges very rapidly from a good starting value, is used. Recently an interior point algorithm was added to the software suite.

CPLEX by CPLEX-Optimization-Inc. (1994) uses a primal-dual logarithmic barrier algorithm (Megiddo 1989) instead with predictor-corrector step (see e.g. Lustig, Marsten and Shanno 1992, Mehrotra and Sun 1992).

MINOS by the Stanford Optimization Laboratory (Murtagh and Saunders 1983) uses a reduced gradient algorithm in conjunction with a quasi-Newton algorithm. The constraints are handled by an active set strategy. Feasibility is maintained throughout the process. On the active constraint manifold, a quasi-Newton approximation is used.

MATLAB: Until recently the matlab QP optimizer delivered only agreeable, although below average performance on classification tasks and was not all too useful for regression tasks (for problems much larger than 100 samples) due to the fact that one is effectively dealing with an optimization problem of size $2 \ell$ where at least half of the eigenvalues of the Hessian vanish. These problems seem to have been addressed in version 5.3 / R11. Matlab now uses interior point codes.

LOQO by Vanderbei (1994) is another example of an interior point code. Section 5.3 discusses the underlying strategies in detail and shows how they can be adapted to SV algorithms.

Maximum margin perceptron by Kowalczyk (2000) is an algorithm specifically tailored to SVs. Unlike most other techniques it works directly in primal space and thus does not have to take the equality constraint on the Lagrange multipliers into account explicitly.

Iterative free set methods The algorithm by Kaufman (Bunch, Kaufman and Parlett 1976, Bunch and Kaufman 1977, 1980, Drucker et al. 1997, Kaufman 1999), uses such a technique starting with all variables on the boundary and adding them as the Karush Kuhn Tucker conditions become more violated. This approach has the advantage of not having to compute the full dot product matrix from the beginning. Instead it is evaluated on the fly, yielding a performance improvement in comparison to tackling the whole optimization problem at once. However, also other algorithms can be modified by subset selection techniques (see Section 5.5) to address this problem.

\subsection{Basic notions}

Most algorithms rely on results from the duality theory in convex optimization. Although we already happened to mention some basic ideas in Section 1.2 we will, for the sake of convenience, briefly review without proof the core results. These are needed in particular to derive an interior point algorithm. For details and proofs (see e.g. Fletcher 1989).

Uniqueness: Every convex constrained optimization problem has a unique minimum. If the problem is strictly convex then the solution is unique. This means that SVs are not plagued with the problem of local minima as Neural Networks are. ${ }^{7}$

Lagrange function: The Lagrange function is given by the primal objective function minus the sum of all products between constraints and corresponding Lagrange multipliers (cf. e.g. Fletcher 1989, Bertsekas 1995). Optimization can be seen as minimzation of the Lagrangian wrt. the primal variables and simultaneous maximization wrt. the Lagrange multipliers, i.e. dual variables. It has a saddle point at the solution. Usually the Lagrange function is only a theoretical device to derive the dual objective function (cf. Section 1.2).

Dual objective function: It is derived by minimizing the Lagrange function with respect to the primal variables and subsequent elimination of the latter. Hence it can be written solely in terms of the dual variables.

Duality gap: For both feasible primal and dual variables the primal objective function (of a convex minimization problem) is always greater or equal than the dual objective function. Since SVMs have only linear constraints the constraint qualifications of the strong duality theorem (Bazaraa, Sherali and Shetty 1993, Theorem 6.2.4) are satisfied and it follows that gap vanishes at optimality. Thus the duality gap is a measure how close (in terms of the objective function) the current set of variables is to the solution.

Karush-Kuhn-Tucker (KKT) conditions: A set of primal and dual variables that is both feasible and satisfies the KKT conditions is the solution (i.e. constraint $\cdot$ dual variable $=0$ ). The sum of the violated KKT terms determines exactly the size of the duality gap (that is, we simply compute the constraint · Lagrangemultiplier part as done in (55)). This allows us to compute the latter quite easily.

A simple intuition is that for violated constraints the dual variable could be increased arbitrarily, thus rendering the Lagrange function arbitrarily large. This, however, is in contradition to the saddlepoint property.

\subsection{Interior point algorithms}

In a nutshell the idea of an interior point algorithm is to compute the dual of the optimization problem (in our case the dual dual of $R_{\mathrm{reg}}[f]$ ) and solve both primal and dual simultaneously. This is done by only gradually enforcing the KKT conditions 
to iteratively find a feasible solution and to use the duality gap between primal and dual objective function to determine the quality of the current set of variables. The special flavour of algorithm we will describe is primal-dual path-following (Vanderbei 1994).

In order to avoid tedious notation we will consider the slightly more general problem and specialize the result to the SVM later. It is understood that unless stated otherwise, variables like $\alpha$ denote vectors and $\alpha_{i}$ denotes its $i$-th component.

$$
\begin{array}{ll}
\text { minimize } & \frac{1}{2} q(\alpha)+\langle c, \alpha\rangle \\
\text { subject to } & A \alpha=b \text { and } l \leq \alpha \leq u
\end{array}
$$

with $c, \alpha, l, u \in \mathbb{R}^{n}, A \in \mathbb{R}^{n \cdot m}, b \in \mathbb{R}^{m}$, the inequalities between vectors holding componentwise and $q(\alpha)$ being a convex function of $\alpha$. Now we will add slack variables to get rid of all inequalities but the positivity constraints. This yields:

$$
\begin{array}{ll}
\text { minimize } & \frac{1}{2} q(\alpha)+\langle c, \alpha\rangle \\
\text { subject to } & A \alpha=b, \alpha-g=l, \alpha+t=u \\
& g, t \geq 0, \alpha \text { free }
\end{array}
$$

The dual of (51) is

$$
\begin{array}{ll}
\text { maximize } & \frac{1}{2}(q(\alpha)-\langle\vec{\partial} q(\alpha), \alpha)\rangle+\langle b, y\rangle+\langle l, z\rangle-\langle u, s\rangle \\
\text { subject to } & \frac{1}{2} \vec{\partial} q(\alpha)+c-(A y)^{\top}+s=z, s, z \geq 0, y \text { free }
\end{array}
$$

Moreover we get the KKT conditions, namely

$$
g_{i} z_{i}=0 \quad \text { and } \quad s_{i} t_{i}=0 \text { for all } i \in[1 \ldots n] .
$$

A necessary and sufficient condition for the optimal solution is that the primal/dual variables satisfy both the feasibility conditions of (51) and (52) and the KKT conditions (53). We proceed to solve (51)-(53) iteratively. The details can be found in Appendix A.

\subsection{Useful tricks}

Before proceeding to further algorithms for quadratic optimization let us briefly mention some useful tricks that can be applied to all algorithms described subsequently and may have significant impact despite their simplicity. They are in part derived from ideas of the interior-point approach.

Training with different regularization parameters: For several reasons (model selection, controlling the number of support vectors, etc.) it may happen that one has to train a SV machine with different regularization parameters $C$, but otherwise rather identical settings. If the parameters $C_{\text {new }}=\tau C_{\text {old }}$ is not too different it is advantageous to use the rescaled values of the Lagrange multipliers (i.e. $\alpha_{i}, \alpha_{i}^{*}$ ) as a starting point for the new optimization problem. Rescaling is necessary to satisfy the modified constraints. One gets

$$
\alpha_{\text {new }}=\tau \alpha_{\text {old }} \quad \text { and likewise } b_{\text {new }}=\tau b_{\text {old }} \text {. }
$$

Assuming that the (dominant) convex part $q(\alpha)$ of the primal objective is quadratic, the $q$ scales with $\tau^{2}$ where as the linear part scales with $\tau$. However, since the linear term dominates the objective function, the rescaled values are still a better starting point than $\alpha=0$. In practice a speedup of approximately $95 \%$ of the overall training time can be observed when using the sequential minimization algorithm, cf. (Smola 1998). A similar reasoning can be applied when retraining with the same regularization parameter but different (yet similar) width parameters of the kernel function. See Cristianini, Campbell and Shawe-Taylor (1998) for details thereon in a different context.

Monitoring convergence via the feasibility gap: In the case of both primal and dual feasible variables the following connection between primal and dual objective function holds:

$$
\text { Dual Obj. = Primal Obj. }-\sum_{i}\left(g_{i} z_{i}+s_{i} t_{i}\right)
$$

This can be seen immediately by the construction of the Lagrange function. In Regression Estimation (with the $\varepsilon$ insensitive loss function) one obtains for $\sum_{i} g_{i} z_{i}+s_{i} t_{i}$

$$
\sum_{i}\left[\begin{array}{l}
+\max \left(0, f\left(x_{i}\right)-\left(y_{i}+\varepsilon_{i}\right)\right)\left(C-\alpha_{i}^{*}\right) \\
-\min \left(0, f\left(x_{i}\right)-\left(y_{i}+\varepsilon_{i}\right)\right) \alpha_{i}^{*} \\
+\max \left(0,\left(y_{i}-\varepsilon_{i}^{*}\right)-f\left(x_{i}\right)\right)\left(C-\alpha_{i}\right) \\
-\min \left(0,\left(y_{i}-\varepsilon_{i}^{*}\right)-f\left(x_{i}\right)\right) \alpha_{i}
\end{array}\right]
$$

Thus convergence with respect to the point of the solution can be expressed in terms of the duality gap. An effective stopping rule is to require

$$
\frac{\sum_{i} g_{i} z_{i}+s_{i} t_{i}}{\mid \text { Primal Objective } \mid+1} \leq \varepsilon_{\text {tol }}
$$

for some precision $\varepsilon_{\text {tol }}$. This condition is much in the spirit of primal dual interior point path following algorithms, where convergence is measured in terms of the number of significant figures (which would be the decimal logarithm of (57)), a convention that will also be adopted in the subsequent parts of this exposition.

\subsection{Subset selection algorithms}

The convex programming algorithms described so far can be used directly on moderately sized (up to 3000) samples datasets without any further modifications. On large datasets, however, it is difficult, due to memory and cpu limitations, to compute the dot product matrix $k\left(x_{i}, x_{j}\right)$ and keep it in memory. A simple calculation shows that for instance storing the dot product matrix of the NIST OCR database (60.000 samples) at single precision would consume 0.7 GBytes. A Cholesky decomposition thereof, which would additionally require roughly the same amount of memory and 64 Teraflops (counting multiplies and adds separately), seems unrealistic, at least at current processor speeds.

A first solution, which was introduced in Vapnik (1982) relies on the observation that the solution can be reconstructed from the SVs alone. Hence, if we knew the SV set beforehand, and 
it fitted into memory, then we could directly solve the reduced problem. The catch is that we do not know the SV set before solving the problem. The solution is to start with an arbitrary subset, a first chunk that fits into memory, train the SV algorithm on it, keep the SVs and fill the chunk up with data the current estimator would make errors on (i.e. data lying outside the $\varepsilon$ tube of the current regression). Then retrain the system and keep on iterating until after training all $K K T$-conditions are satisfied.

The basic chunking algorithm just postponed the underlying problem of dealing with large datasets whose dot-product matrix cannot be kept in memory: it will occur for larger training set sizes than originally, but it is not completely avoided. Hence the solution is Osuna, Freund and Girosi (1997) to use only a subset of the variables as a working set and optimize the problem with respect to them while freezing the other variables. This method is described in detail in Osuna, Freund and Girosi (1997), Joachims (1999) and Saunders et al. (1998) for the case of pattern recognition. ${ }^{8}$

An adaptation of these techniques to the case of regression with convex cost functions can be found in Appendix B. The basic structure of the method is described by Algorithm 1 .

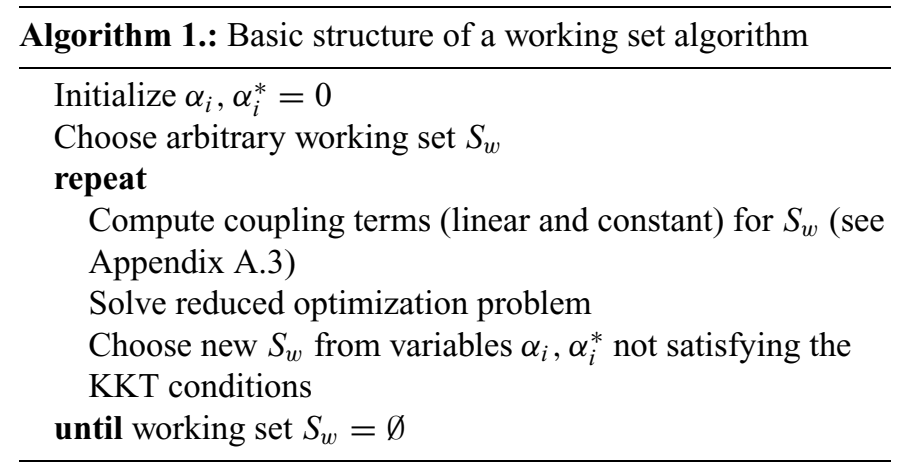

\subsection{Sequential minimal optimization}

Recently an algorithm-Sequential Minimal Optimization (SMO) - was proposed (Platt 1999) that puts chunking to the extreme by iteratively selecting subsets only of size 2 and optimizing the target function with respect to them. It has been reported to have good convergence properties and it is easily implemented. The key point is that for a working set of 2 the optimization subproblem can be solved analytically without explicitly invoking a quadratic optimizer.

While readily derived for pattern recognition by Platt (1999), one simply has to mimick the original reasoning to obtain an extension to Regression Estimation. This is what will be done in Appendix $\mathrm{C}$ (the pseudocode can be found in Smola and Schölkopf (1998b)). The modifications consist of a pattern dependent regularization, convergence control via the number of significant figures, and a modified system of equations to solve the optimization problem in two variables for regression analytically.

Note that the reasoning only applies to SV regression with the $\varepsilon$ insensitive loss function-for most other convex cost func- tions an explicit solution of the restricted quadratic programming problem is impossible. Yet, one could derive an analogous nonquadratic convex optimization problem for general cost functions but at the expense of having to solve it numerically.

The exposition proceeds as follows: first one has to derive the (modified) boundary conditions for the constrained 2 indices $(i, j)$ subproblem in regression, next one can proceed to solve the optimization problem analytically, and finally one has to check, which part of the selection rules have to be modified to make the approach work for regression. Since most of the content is fairly technical it has been relegated to Appendix C.

The main difference in implementations of SMO for regression can be found in the way the constant offset $b$ is determined (Keerthi et al. 1999) and which criterion is used to select a new set of variables. We present one such strategy in Appendix C.3. However, since selection strategies are the focus of current research we recommend that readers interested in implementing the algorithm make sure they are aware of the most recent developments in this area.

Finally, we note that just as we presently describe a generalization of SMO to regression estimation, other learning problems can also benefit from the underlying ideas. Recently, a SMO algorithm for training novelty detection systems (i.e. one-class classification) has been proposed (Schölkopf et al. 2001).

\section{Variations on a theme}

There exists a large number of algorithmic modifications of the SV algorithm, to make it suitable for specific settings (inverse problems, semiparametric settings), different ways of measuring capacity and reductions to linear programming (convex combinations) and different ways of controlling capacity. We will mention some of the more popular ones.

\subsection{Convex combinations and $\ell_{1}$-norms}

All the algorithms presented so far involved convex, and at best, quadratic programming. Yet one might think of reducing the problem to a case where linear programming techniques can be applied. This can be done in a straightforward fashion (Mangasarian 1965, 1968, Weston et al. 1999, Smola, Schölkopf and Rätsch 1999) for both SV pattern recognition and regression. The key is to replace (35) by

$$
R_{\mathrm{reg}}[f]:=R_{\mathrm{emp}}[f]+\lambda\|\alpha\|_{1}
$$

where $\|\alpha\|_{1}$ denotes the $\ell_{1}$ norm in coefficient space. Hence one uses the SV kernel expansion (11)

$$
f(x)=\sum_{i=1}^{\ell} \alpha_{i} k\left(x_{i}, x\right)+b
$$

with a different way of controlling capacity by minimizing

$$
R_{\mathrm{reg}}[f]=\frac{1}{\ell} \sum_{i=1}^{\ell} c\left(x_{i}, y_{i}, f\left(x_{i}\right)\right)+\lambda \sum_{i=1}^{\ell}\left|\alpha_{i}\right| .
$$


For the $\varepsilon$-insensitive loss function this leads to a linear programming problem. In the other cases, however, the problem still stays a quadratic or general convex one, and therefore may not yield the desired computational advantage. Therefore we will limit ourselves to the derivation of the linear programming problem in the case of $|\cdot|_{\varepsilon}$ cost function. Reformulating (59) yields

$$
\begin{aligned}
\operatorname{minimize} & \sum_{i=1}^{\ell}\left(\alpha_{i}+\alpha_{i}^{*}\right)+C \sum_{i=1}^{\ell}\left(\xi_{i}+\xi_{i}^{*}\right) \\
\text { subject to } & \left\{\begin{aligned}
y_{i}-\sum_{j=1}^{\ell}\left(\alpha_{j}-\alpha_{j}^{*}\right) k\left(x_{j}, x_{i}\right)-b \leq \varepsilon+\xi_{i} \\
\sum_{j=1}^{\ell}\left(\alpha_{j}-\alpha_{j}^{*}\right) k\left(x_{j}, x_{i}\right)+b-y_{i} \leq \varepsilon+\xi_{i}^{*} \\
\alpha_{i}, \alpha_{i}^{*}, \xi_{i}, \xi_{i}^{*} \geq 0
\end{aligned}\right.
\end{aligned}
$$

Unlike in the classical SV case, the transformation into its dual does not give any improvement in the structure of the optimization problem. Hence it is best to minimize $R_{\text {reg }}[f]$ directly, which can be achieved by a linear optimizer, (e.g. Dantzig 1962, Lustig, Marsten and Shanno 1990, Vanderbei 1997).

In (Weston et al. 1999) a similar variant of the linear SV approach is used to estimate densities on a line. One can show (Smola et al. 2000) that one may obtain bounds on the generalization error which exhibit even better rates (in terms of the entropy numbers) than the classical SV case (Williamson, Smola and Schölkopf 1998).

\subsection{Automatic tuning of the insensitivity tube}

Besides standard model selection issues, i.e. how to specify the trade-off between empirical error and model capacity there also exists the problem of an optimal choice of a cost function. In particular, for the $\varepsilon$-insensitive cost function we still have the problem of choosing an adequate parameter $\varepsilon$ in order to achieve good performance with the SV machine.

Smola et al. (1998a) show the existence of a linear dependency between the noise level and the optimal $\varepsilon$-parameter for $\mathrm{SV}$ regression. However, this would require that we know something about the noise model. This knowledge is not available in general. Therefore, albeit providing theoretical insight, this finding by itself is not particularly useful in practice. Moreover, if we really knew the noise model, we most likely would not choose the $\varepsilon$-insensitive cost function but the corresponding maximum likelihood loss function instead.

There exists, however, a method to construct SV machines that automatically adjust $\varepsilon$ and moreover also, at least asymptotically, have a predetermined fraction of sampling points as SVS (Schölkopf et al. 2000). We modify (35) such that $\varepsilon$ becomes a variable of the optimization problem, including an extra term in the primal objective function which attempts to minimize $\varepsilon$. In other words

$$
\operatorname{minimize} R_{v}[f]:=R_{\mathrm{emp}}[f]+\frac{\lambda}{2}\|w\|^{2}+v \varepsilon
$$

for some $v>0$. Hence (42) becomes (again carrying out the usual transformation between $\lambda, \ell$ and $C$ )

$$
\begin{array}{ll}
\operatorname{minimize} & \frac{1}{2}\|w\|^{2}+C\left(\sum_{i=1}^{\ell}\left(\tilde{c}\left(\xi_{i}\right)+\tilde{c}\left(\xi_{i}^{*}\right)\right)+\ell v \varepsilon\right) \\
\text { subject to } \quad\left\{\begin{array}{l}
y_{i}-\left\langle w, x_{i}\right\rangle-b \leq \varepsilon+\xi_{i} \\
\left\langle w, x_{i}\right\rangle+b-y_{i} \leq \varepsilon+\xi_{i}^{*} \\
\xi_{i}, \xi_{i}^{*} \geq 0
\end{array}\right.
\end{array}
$$

We consider the standard $|\cdot|_{\varepsilon}$ loss function. Computing the dual of (62) yields

$$
\begin{array}{ll}
\operatorname{maximize} & \left\{\begin{array}{l}
-\frac{1}{2} \sum_{i, j=1}^{\ell}\left(\alpha_{i}-\alpha_{i}^{*}\right)\left(\alpha_{j}-\alpha_{j}^{*}\right) k\left(x_{i}, x_{j}\right) \\
+\sum_{i=1}^{\ell} y_{i}\left(\alpha_{i}-\alpha_{i}^{*}\right)
\end{array}\right. \\
\text { subject to }\left\{\begin{array}{l}
\sum_{i=1}^{\ell}\left(\alpha_{i}-\alpha_{i}^{*}\right)=0 \\
\sum_{i=1}^{\ell}\left(\alpha_{i}+\alpha_{i}^{*}\right) \leq C v \ell \\
\alpha_{i}, \alpha_{i}^{*} \quad \in[0, C]
\end{array}\right.
\end{array}
$$

Note that the optimization problem is thus very similar to the $\varepsilon$ SV one: the target function is even simpler (it is homogeneous), but there is an additional constraint. For information on how this affects the implementation (cf. Chang and Lin 2001).

Besides having the advantage of being able to automatically determine $\varepsilon$ (63) also has another advantage. It can be used to pre-specify the number of SVs:

\section{Theorem 9 (Schölkopf et al. 2000).}

1. $v$ is an upper bound on the fraction of errors.

2. $v$ is a lower bound on the fraction of SVS.

3. Suppose the data has been generated iid from a distribution $p(x, y)=p(x) p(y \mid x)$ with a continuous conditional distribution $p(y \mid x)$. With probability 1 , asymptotically, v equals the fraction of $S V S$ and the fraction of errors.

Essentially, $\nu$-SV regression improves upon $\varepsilon$-SV regression by allowing the tube width to adapt automatically to the data. What is kept fixed up to this point, however, is the shape of the tube. One can, however, go one step further and use parametric tube models with non-constant width, leading to almost identical optimization problems (Schölkopf et al. 2000).

Combining $v$-SV regression with results on the asymptotical optimal choice of $\varepsilon$ for a given noise model (Smola et al. 1998a) leads to a guideline how to adjust $v$ provided the class of noise models (e.g. Gaussian or Laplacian) is known.

Remark 10 (Optimal choice of $v$ ). Denote by $\mathfrak{p}$ a probability density with unit variance, and by $\mathfrak{P}$ a famliy of noise models generated from $\mathfrak{p}$ by $\mathfrak{P}:=\left\{p \mid p=\frac{1}{\sigma} \mathfrak{p}\left(\frac{y}{\sigma}\right)\right\}$. Moreover assume 


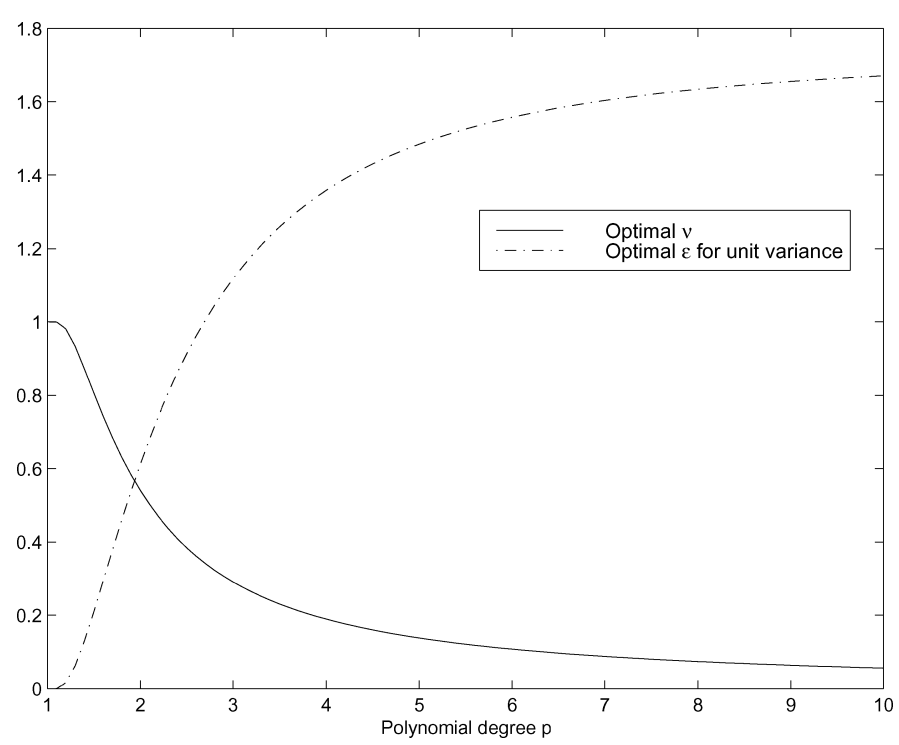

Fig. 5. Optimal $v$ and $\varepsilon$ for various degrees of polynomial additive noise

that the data were drawn iid from $p(x, y)=p(x) p(y-f(x))$ with $p(y-f(x))$ continuous. Then under the assumption of uniform convergence, the asymptotically optimal value of $v$ is

$$
v=1-\int_{-\varepsilon}^{\varepsilon} \mathfrak{p}(t) d t
$$

where $\quad \varepsilon:=\underset{\tau}{\operatorname{argmin}}(\mathfrak{p}(-\tau)+\mathfrak{p}(\tau))^{-2}\left(1-\int_{-\tau}^{\tau} \mathfrak{p}(t) d t\right)$

For polynomial noise models, i.e. densities of type $\exp \left(-|\xi|^{p}\right)$ one may compute the corresponding (asymptotically) optimal values of $v$. They are given in Fig. 5. For further details see (Schölkopf et al. 2000, Smola 1998); an experimental validation has been given by Chalimourda, Schölkopf and Smola (2000).

We conclude this section by noting that $\nu$-SV regression is related to the idea of trimmed estimators. One can show that the regression is not influenced if we perturb points lying outside the tube. Thus, the regression is essentially computed by discarding a certain fraction of outliers, specified by $v$, and computing the regression estimate from the remaining points (Schölkopf et al. 2000).

\section{Regularization}

So far we were not concerned about the specific properties of the map $\Phi$ into feature space and used it only as a convenient trick to construct nonlinear regression functions. In some cases the map was just given implicitly by the kernel, hence the map itself and many of its properties have been neglected. A deeper understanding of the kernel map would also be useful to choose appropriate kernels for a specific task (e.g. by incorporating prior knowledge (Schölkopf et al. 1998a)). Finally the feature map seems to defy the curse of dimensionality (Bellman 1961) by making problems seemingly easier yet reliable via a map into some even higher dimensional space.

In this section we focus on the connections between SV methods and previous techniques like Regularization Networks (Girosi, Jones and Poggio 1993). ${ }^{9}$ In particular we will show that SV machines are essentially Regularization Networks (RN) with a clever choice of cost functions and that the kernels are Green's function of the corresponding regularization operators. For a full exposition of the subject the reader is referred to Smola, Schölkopf and Müller (1998c).

\subsection{Regularization networks}

Let us briefly review the basic concepts of RNs. As in (35) we minimize a regularized risk functional. However, rather than enforcing flatness in feature space we try to optimize some smoothness criterion for the function in input space. Thus we get

$$
R_{\mathrm{reg}}[f]:=R_{\mathrm{emp}}[f]+\frac{\lambda}{2}\|P f\|^{2} .
$$

Here $P$ denotes a regularization operator in the sense of Tikhonov and Arsenin (1977), i.e. $P$ is a positive semidefinite operator mapping from the Hilbert space $H$ of functions $f$ under consideration to a dot product space $D$ such that the expression $\langle P f \cdot P g\rangle$ is well defined for $f, g \in H$. For instance by choosing a suitable operator that penalizes large variations of $f$ one can reduce the well-known overfitting effect. Another possible setting also might be an operator $P$ mapping from $L^{2}\left(\mathbb{R}^{n}\right)$ into some Reproducing Kernel Hilbert Space (RKHS) (Aronszajn, 1950, Kimeldorf and Wahba 1971, Saitoh 1988, Schölkopf 1997 , Girosi 1998).

Using an expansion of $f$ in terms of some symmetric function $k\left(\mathbf{x}_{i}, \mathbf{x}_{j}\right)$ (note here, that $k$ need not fulfill Mercer's condition and can be chosen arbitrarily since it is not used to define a regularization term),

$$
f(\mathbf{x})=\sum_{i=1}^{\ell} \alpha_{i} k\left(x_{i}, x\right)+b
$$

and the $\varepsilon$-insensitive cost function, this leads to a quadratic programming problem similar to the one for SVs. Using

$$
D_{i j}:=\left\langle(P k)\left(x_{i}, .\right) \cdot(P k)\left(x_{j}, .\right)\right\rangle
$$

we get $\alpha=D^{-1} K\left(\beta-\beta^{*}\right)$, with $\beta, \beta^{*}$ being the solution of

$$
\begin{array}{ll}
\operatorname{minimize} & \frac{1}{2}\left(\beta^{*}-\beta\right)^{\top} K D^{-1} K\left(\beta^{*}-\beta\right) \\
& -\left(\beta^{*}-\beta\right)^{\top} y-\varepsilon \sum_{i=1}^{\ell}\left(\beta_{i}+\beta_{i}^{*}\right) \\
\text { subject to } & \sum_{i=1}^{\ell}\left(\beta_{i}-\beta_{i}^{*}\right)=0 \quad \text { and } \beta_{i}, \beta_{i}^{*} \in[0, C] .
\end{array}
$$


Unfortunately, this setting of the problem does not preserve sparsity in terms of the coefficients, as a potentially sparse decomposition in terms of $\beta_{i}$ and $\beta_{i}^{*}$ is spoiled by $D^{-1} K$, which is not in general diagonal.

\subsection{Green's functions}

Comparing (10) with (67) leads to the question whether and under which condition the two methods might be equivalent and therefore also under which conditions regularization networks might lead to sparse decompositions, i.e. only a few of the expansion coefficients $\alpha_{i}$ in $f$ would differ from zero. A sufficient condition is $D=K$ and thus $K D^{-1} K=K$ (if $K$ does not have full rank we only need that $K D^{-1} K=K$ holds on the image of $K)$ :

$$
k\left(x_{i}, x_{j}\right)=\left\langle(P k)\left(x_{i}, .\right) \cdot(P k)\left(x_{j}, .\right)\right\rangle
$$

Our goal now is to solve the following two problems:

1. Given a regularization operator $P$, find a kernel $k$ such that a SV machine using $k$ will not only enforce flatness in feature space, but also correspond to minimizing a regularized risk functional with $P$ as regularizer.

2. Given an SV kernel $k$, find a regularization operator $P$ such that a SV machine using this kernel can be viewed as a Regularization Network using $P$.

These two problems can be solved by employing the concept of Green's functions as described in Girosi, Jones and Poggio (1993). These functions were introduced for the purpose of solving differential equations. In our context it is sufficient to know that the Green's functions $G_{x_{i}}(x)$ of $P^{*} P$ satisfy

$$
\left(P^{*} P G_{x_{i}}\right)(x)=\delta_{x_{i}}(x) .
$$

Here, $\delta_{x_{i}}(x)$ is the $\delta$-distribution (not to be confused with the Kronecker symbol $\delta_{i j}$ ) which has the property that $\left\langle f \cdot \delta_{x_{i}}\right\rangle=f\left(x_{i}\right)$. The relationship between kernels and regularization operators is formalized in the following proposition:

Proposition 1 (Smola, Schölkopf and Müller 1998b). Let $P$ be a regularization operator, and $G$ be the Green's function of $P^{*} P$. Then $G$ is a Mercer Kernel such that $D=K$. SV machines using $G$ minimize risk functional (64) with $P$ as regularization operator.

In the following we will exploit this relationship in both ways: to compute Green's functions for a given regularization operator $P$ and to infer the regularizer, given a kernel $k$.

\subsection{Translation invariant kernels}

Let us now more specifically consider regularization operators $\hat{P}$ that may be written as multiplications in Fourier space

$$
\langle P f \cdot P g\rangle=\frac{1}{(2 \pi)^{n / 2}} \int_{\Omega} \frac{\overline{\tilde{f}(\omega)} \tilde{g}(\omega)}{P(\omega)} d \omega
$$

with $\tilde{f}(\omega)$ denoting the Fourier transform of $f(x)$, and $P(\omega)=$ $P(-\omega)$ real valued, nonnegative and converging to 0 for $|\omega| \rightarrow$ $\infty$ and $\Omega:=\operatorname{supp}[P(\omega)]$. Small values of $P(\omega)$ correspond to a strong attenuation of the corresponding frequencies. Hence small values of $P(\omega)$ for large $\omega$ are desirable since high frequency components of $\tilde{f}$ correspond to rapid changes in $f$. $P(\omega)$ describes the filter properties of $P^{*} P$. Note that no attenuation takes place for $P(\omega)=0$ as these frequencies have been excluded from the integration domain.

For regularization operators defined in Fourier Space by (70) one can show by exploiting $P(\omega)=P(-\omega)=\overline{P(\omega)}$ that

$$
G\left(x_{i}, x\right)=\frac{1}{(2 \pi)^{n / 2}} \int_{\mathbb{R}^{n}} e^{i \omega\left(x_{i}-x\right)} P(\omega) d \omega
$$

is a corresponding Green's function satisfying translational invariance, i.e.

$$
G\left(x_{i}, x_{j}\right)=G\left(x_{i}-x_{j}\right) \quad \text { and } \quad \tilde{G}(\omega)=P(\omega) .
$$

This provides us with an efficient tool for analyzing SV kernels and the types of capacity control they exhibit. In fact the above is a special case of Bochner's theorem (Bochner 1959) stating that the Fourier transform of a positive measure constitutes a positive Hilbert Schmidt kernel.

Example 2 (Gaussian kernels). Following the exposition of Yuille and Grzywacz (1988) as described in Girosi, Jones and Poggio (1993), one can see that for

$$
\|P f\|^{2}=\int d x \sum_{m} \frac{\sigma^{2 m}}{m ! 2^{m}}\left(\hat{O}^{m} f(x)\right)^{2}
$$

with $\hat{O}^{2 m}=\Delta^{m}$ and $\hat{O}^{2 m+1}=\nabla \Delta^{m}, \Delta$ being the Laplacian and $\nabla$ the Gradient operator, we get Gaussians kernels (31). Moreover, we can provide an equivalent representation of $P$ in terms of its Fourier properties, i.e. $P(\omega)=e^{-\frac{\sigma^{2}\|\omega\|^{2}}{2}}$ up to a multiplicative constant.

Training an SV machine with Gaussian RBF kernels (Schölkopf et al. 1997) corresponds to minimizing the specific cost function with a regularization operator of type (73). Recall that (73) means that all derivatives of $f$ are penalized (we have a pseudodifferential operator) to obtain a very smooth estimate. This also explains the good performance of SV machines in this case, as it is by no means obvious that choosing a flat function in some high dimensional space will correspond to a simple function in low dimensional space, as shown in Smola, Schölkopf and Müller (1998c) for Dirichlet kernels.

The question that arises now is which kernel to choose. Let us think about two extreme situations.

1. Suppose we already knew the shape of the power spectrum $\operatorname{Pow}(\omega)$ of the function we would like to estimate. In this case we choose $k$ such that $\tilde{k}$ matches the power spectrum (Smola 1998).

2. If we happen to know very little about the given data a general smoothness assumption is a reasonable choice. Hence 
we might want to choose a Gaussian kernel. If computing time is important one might moreover consider kernels with compact support, e.g. using the $B_{q}$-spline kernels (cf. (32)). This choice will cause many matrix elements $k_{i j}=k\left(x_{i}-x_{j}\right)$ to vanish.

The usual scenario will be in between the two extreme cases and we will have some limited prior knowledge available. For more information on using prior knowledge for choosing kernels (see Schölkopf et al. 1998a).

\subsection{Capacity control}

All the reasoning so far was based on the assumption that there exist ways to determine model parameters like the regularization constant $\lambda$ or length scales $\sigma$ of rbf-kernels. The model selection issue itself would easily double the length of this review and moreover it is an area of active and rapidly moving research. Therefore we limit ourselves to a presentation of the basic concepts and refer the interested reader to the original publications.

It is important to keep in mind that there exist several fundamentally different approaches such as Minimum Description Length (cf. e.g. Rissanen 1978, Li and Vitányi 1993) which is based on the idea that the simplicity of an estimate, and therefore also its plausibility is based on the information (number of bits) needed to encode it such that it can be reconstructed.

Bayesian estimation, on the other hand, considers the posterior probability of an estimate, given the observations $X=$ $\left\{\left(x_{1}, y_{1}\right), \ldots\left(x_{\ell}, y_{\ell}\right)\right\}$, an observation noise model, and a prior probability distribution $p(f)$ over the space of estimates (parameters). It is given by Bayes Rule $p(f \mid X) p(X)=$ $p(X \mid f) p(f)$. Since $p(X)$ does not depend on $f$, one can maximize $p(X \mid f) p(f)$ to obtain the so-called MAP estimate. ${ }^{10}$ As a rule of thumb, to translate regularized risk functionals into Bayesian MAP estimation schemes, all one has to do is to consider $\exp \left(-R_{\text {reg }}[f]\right)=p(f \mid X)$. For a more detailed discussion (see e.g. Kimeldorf and Wahba 1970, MacKay 1991, Neal 1996, Rasmussen 1996, Williams 1998).

A simple yet powerful way of model selection is cross validation. This is based on the idea that the expectation of the error on a subset of the training sample not used during training is identical to the expected error itself. There exist several strategies such as 10 -fold crossvalidation, leave-one out error $(\ell$-fold crossvalidation), bootstrap and derived algorithms to estimate the crossvalidation error itself(see e.g. Stone 1974, Wahba 1980, Efron 1982, Efron and Tibshirani 1994, Wahba 1999, Jaakkola and Haussler 1999) for further details.

Finally, one may also use uniform convergence bounds such as the ones introduced by Vapnik and Chervonenkis (1971). The basic idea is that one may bound with probability $1-\eta$ (with $\eta>0)$ the expected risk $R[f]$ by $R_{\text {emp }}[f]+\Phi(\mathcal{F}, \eta)$, where $\Phi$ is a confidence term depending on the class of functions $\mathcal{F}$. Several criteria for measuring the capacity of $\mathcal{F}$ exist, such as the $V C$-Dimension which, in pattern recognition problems, is given by the maximum number of points that can be separated by the function class in all possible ways, the Covering Number which is the number of elements from $\mathcal{F}$ that are needed to cover $\mathcal{F}$ with accuracy of at least $\varepsilon$, Entropy Numbers which are the functional inverse of Covering Numbers, and many more variants thereof (see e.g. Vapnik 1982, 1998, Devroye, Györfi and Lugosi 1996, Williamson, Smola and Schölkopf 1998, Shawe-Taylor et al. 1998).

\section{Conclusion}

Due to the already quite large body of work done in the field of $\mathrm{SV}$ research it is impossible to write a tutorial on SV regression which includes all contributions to this field. This also would be quite out of the scope of a tutorial and rather be relegated to textbooks on the matter (see Schölkopf and Smola (2002) for a comprehensive overview, Schölkopf, Burges and Smola (1999a) for a snapshot of the current state of the art, Vapnik (1998) for an overview on statistical learning theory, or Cristianini and ShaweTaylor (2000) for an introductory textbook). Still the authors hope that this work provides a not overly biased view of the state of the art in SV regression research. We deliberately omitted (among others) the following topics.

\subsection{Missing topics}

Mathematical programming: Starting from a completely different perspective algorithms have been developed that are similar in their ideas to SV machines. A good primer might be (Bradley, Fayyad and Mangasarian 1998). (Also see Mangasarian 1965, 1969, Street and Mangasarian 1995). A comprehensive discussion of connections between mathematical programming and SV machines has been given by (Bennett 1999).

Density estimation: with SV machines (Weston et al. 1999, Vapnik 1999). There one makes use of the fact that the cumulative distribution function is monotonically increasing, and that its values can be predicted with variable confidence which is adjusted by selecting different values of $\varepsilon$ in the loss function.

Dictionaries: were originally introduced in the context of wavelets by (Chen, Donoho and Saunders 1999) to allow for a large class of basis functions to be considered simultaneously, e.g. kernels with different widths. In the standard SV case this is hardly possible except by defining new kernels as linear combinations of differently scaled ones: choosing the regularization operator already determines the kernel completely (Kimeldorf and Wahba 1971, Cox and O'Sullivan 1990, Schölkopf et al. 2000). Hence one has to resort to linear programming (Weston et al. 1999).

Applications: The focus of this review was on methods and theory rather than on applications. This was done to limit the size of the exposition. State of the art, or even record performance was reported in Müller et al. (1997), Drucker et al. (1997), Stitson et al. (1999) and Mattera and Haykin (1999). 
In many cases, it may be possible to achieve similar performance with neural network methods, however, only if many parameters are optimally tuned by hand, thus depending largely on the skill of the experimenter. Certainly, SV machines are not a "silver bullet." However, as they have only few critical parameters (e.g. regularization and kernel width), state-of-the-art results can be achieved with relatively little effort.

\subsection{Open issues}

Being a very active field there exist still a number of open issues that have to be addressed by future research. After that the algorithmic development seems to have found a more stable stage, one of the most important ones seems to be to find tight error bounds derived from the specific properties of kernel functions. It will be of interest in this context, whether SV machines, or similar approaches stemming from a linear programming regularizer, will lead to more satisfactory results.

Moreover some sort of "luckiness framework" (Shawe-Taylor et al. 1998) for multiple model selection parameters, similar to multiple hyperparameters and automatic relevance detection in Bayesian statistics (MacKay 1991, Bishop 1995), will have to be devised to make SV machines less dependent on the skill of the experimenter.

It is also worth while to exploit the bridge between regularization operators, Gaussian processes and priors (see e.g. (Williams 1998)) to state Bayesian risk bounds for SV machines in order to compare the predictions with the ones from VC theory. Optimization techniques developed in the context of SV machines also could be used to deal with large datasets in the Gaussian process settings.

Prior knowledge appears to be another important question in $\mathrm{SV}$ regression. Whilst invariances could be included in pattern recognition in a principled way via the virtual SV mechanism and restriction of the feature space (Burges and Schölkopf 1997, Schölkopf et al. 1998a), it is still not clear how (probably) more subtle properties, as required for regression, could be dealt with efficiently.

Reduced set methods also should be considered for speeding up prediction (and possibly also training) phase for large datasets (Burges and Schölkopf 1997, Osuna and Girosi 1999, Schölkopf et al. 1999b, Smola and Schölkopf 2000). This topic is of great importance as data mining applications require algorithms that are able to deal with databases that are often at least one order of magnitude larger ( 1 million samples) than the current practical size for SV regression.

Many more aspects such as more data dependent generalization bounds, efficient training algorithms, automatic kernel selection procedures, and many techniques that already have made their way into the standard neural networks toolkit, will have to be considered in the future.

Readers who are tempted to embark upon a more detailed exploration of these topics, and to contribute their own ideas to this exciting field, may find it useful to consult the web page www.kernel-machines.org.

\section{Appendix A: Solving the interior-point equations}

\section{A.1. Path following}

Rather than trying to satisfy (53) directly we will solve a modified version thereof for some $\mu>0$ substituted on the rhs in the first place and decrease $\mu$ while iterating.

$$
g_{i} z_{i}=\mu, \quad s_{i} t_{i}=\mu \quad \text { for all } i \in[1 \ldots n] .
$$

Still it is rather difficult to solve the nonlinear system of equations (51), (52), and (74) exactly. However we are not interested in obtaining the exact solution to the approximation (74). Instead, we seek a somewhat more feasible solution for a given $\mu$, then decrease $\mu$ and repeat. This can be done by linearizing the above system and solving the resulting equations by a predictorcorrector approach until the duality gap is small enough. The advantage is that we will get approximately equal performance as by trying to solve the quadratic system directly, provided that the terms in $\Delta^{2}$ are small enough.

$$
\begin{aligned}
& A(\alpha+\Delta \alpha)=b \\
& \alpha+\Delta \alpha-g-\Delta g=l \\
& \alpha+\Delta \alpha+t+\Delta t=u \\
& c+\frac{1}{2} \partial_{\alpha} q(\alpha)+\frac{1}{2} \partial_{\alpha}^{2} q(\alpha) \Delta \alpha-(A(y+\Delta y))^{\top} \\
& \quad+s+\Delta s=z+\Delta z \\
& \left(g_{i}+\Delta g_{i}\right)\left(z_{i}+\Delta z_{i}\right)=\mu \\
& \left(s_{i}+\Delta s_{i}\right)\left(t_{i}+\Delta t_{i}\right)=\mu
\end{aligned}
$$

Solving for the variables in $\Delta$ we get

$$
\begin{aligned}
& A \Delta \alpha=b-A \alpha=: \rho \\
& \Delta \alpha-\Delta g=l-\alpha+g=: v \\
& \Delta \alpha+\Delta t=u-\alpha-t=: \tau \\
& (A \Delta y)^{\top}+\Delta z-\Delta s-\frac{1}{2} \partial_{\alpha}^{2} q(\alpha) \Delta \alpha \\
& \quad=c-(A y)^{\top}+s-z+\frac{1}{2} \partial_{\alpha} q(\alpha)=: \sigma \\
& g^{-1} z \Delta g+\Delta z=\mu g^{-1}-z-g^{-1} \Delta g \Delta z=: \gamma_{z} \\
& t^{-1} s \Delta t+\Delta s=\mu t^{-1}-s-t^{-1} \Delta t \Delta s=: \gamma_{s}
\end{aligned}
$$

where $g^{-1}$ denotes the vector $\left(1 / g_{1}, \ldots, 1 / g_{n}\right)$, and $t$ analogously. Moreover denote $g^{-1} z$ and $t^{-1} s$ the vector generated by the componentwise product of the two vectors. Solving for 
$\Delta g, \Delta t, \Delta z, \Delta s$ we get

$$
\begin{array}{rlrl}
\Delta g=z^{-1} g\left(\gamma_{z}-\Delta z\right) & \Delta z & =g^{-1} z(\hat{v}-\Delta \alpha) \\
\Delta t=s^{-1} t\left(\gamma_{s}-\Delta s\right) & \Delta s & =t^{-1} s(\Delta \alpha-\hat{\tau}) \\
\text { where } & \hat{v} & :=v-z^{-1} g \gamma_{z} \\
\hat{\tau} & :=\tau-s^{-1} t \gamma_{s}
\end{array}
$$

Now we can formulate the reduced KKT-system (see (Vanderbei 1994) for the quadratic case):

$$
\left[\begin{array}{cc}
-H & A^{\top} \\
A & 0
\end{array}\right]\left[\begin{array}{l}
\Delta \alpha \\
\Delta y
\end{array}\right]=\left[\begin{array}{c}
\sigma-g^{-1} z \hat{v}-t^{-1} s \hat{\tau} \\
\rho
\end{array}\right]
$$

where $H:=\left(\frac{1}{2} \partial_{\alpha}^{2} q(\alpha)+g^{-1} z+t^{-1} s\right)$.

\section{A.2. Iteration strategies}

For the predictor-corrector method we proceed as follows. In the predictor step solve the system of (75) and (76) with $\mu=0$ and all $\Delta$-terms on the rhs set to 0 , i.e. $\gamma_{z}=z, \gamma_{s}=s$. The values in $\Delta$ are substituted back into the definitions for $\gamma_{z}$ and $\gamma_{s}$ and (75) and (76) are solved again in the corrector step. As the quadratic part in (76) is not affected by the predictor-corrector steps, we only need to invert the quadratic matrix once. This is done best by manually pivoting for the $H$ part, as it is positive definite.

Next the values in $\Delta$ obtained by such an iteration step are used to update the corresponding values in $\alpha, s, t, z, \ldots$. To ensure that the variables meet the positivity constraints, the steplength $\xi$ is chosen such that the variables move at most $1-\varepsilon$ of their initial distance to the boundaries of the positive orthant. Usually (Vanderbei 1994) one sets $\varepsilon=0.05$.

Another heuristic is used for computing $\mu$, the parameter determining how much the KKT-conditions should be enforced. Obviously it is our aim to reduce $\mu$ as fast as possible, however if we happen to choose it too small, the condition of the equations will worsen drastically. A setting that has proven to work robustly is

$$
\mu=\frac{\langle g, z\rangle+\langle s, t\rangle}{2 n}\left(\frac{\xi-1}{\xi+10}\right)^{2} .
$$

The rationale behind (77) is to use the average of the satisfaction of the KKT conditions (74) as point of reference and then decrease $\mu$ rapidly if we are far enough away from the boundaries of the positive orthant, to which all variables (except $y$ ) are constrained to.

Finally one has to come up with good initial values. Analogously to Vanderbei (1994) we choose a regularized version of (76) in order to determine the initial conditions. One solves

$$
\left[\begin{array}{cc}
-\left(\frac{1}{2} \partial_{\alpha}^{2} q(\alpha)+\mathbf{1}\right) & A^{\top} \\
A & \mathbf{1}
\end{array}\right]\left[\begin{array}{l}
\alpha \\
y
\end{array}\right]=\left[\begin{array}{l}
c \\
b
\end{array}\right]
$$

and subsequently restricts the solution to a feasible set

$$
\begin{aligned}
x & =\max \left(x, \frac{u}{100}\right) \\
g & =\min (\alpha-l, u) \\
t & =\min (u-\alpha, u) \\
z & =\min \left(\Theta\left(\frac{1}{2} \partial_{\alpha} q(\alpha)+c-(A y)^{\top}\right)+\frac{u}{100}, u\right) \\
s & =\min \left(\Theta\left(-\frac{1}{2} \partial_{\alpha} q(\alpha)-c+(A y)^{\top}\right)+\frac{u}{100}, u\right)
\end{aligned}
$$

$\Theta(\cdot)$ denotes the Heavyside function, i.e. $\Theta(x)=1$ for $x>0$ and $\Theta(x)=0$ otherwise.

\section{A.3. Special considerations for $S V$ regression}

The algorithm described so far can be applied to both SV pattern recognition and regression estimation. For the standard setting in pattern recognition we have

$$
q(\alpha)=\sum_{i, j=0}^{\ell} \alpha_{i} \alpha_{j} y_{i} y_{j} k\left(x_{i}, x_{j}\right)
$$

and consequently $\partial_{\alpha_{i}} q(\alpha)=0, \partial_{\alpha_{i} \alpha_{i}}^{2} q(\alpha)=y_{i} y_{j} k\left(x_{i}, x_{j}\right)$, i.e. the Hessian is dense and the only thing we can do is compute its Cholesky factorization to compute (76). In the case of SV regression, however we have (with $\left.\alpha:=\left(\alpha_{1}, \ldots, \alpha_{\ell}, \alpha_{1}^{*}, \ldots, \alpha_{\ell}^{*}\right)\right)$

$$
\begin{aligned}
q(\alpha)= & \sum_{i, j=1}^{\ell}\left(\alpha_{i}-\alpha_{i}^{*}\right)\left(\alpha_{j}-\alpha_{j}^{*}\right) k\left(x_{i}, x_{j}\right) \\
& +2 C \sum_{i=1}^{\ell} T\left(\alpha_{i}\right)+T\left(\alpha_{i}^{*}\right)
\end{aligned}
$$

and therefore

$$
\begin{aligned}
\partial_{\alpha_{i}} q(\alpha) & =\frac{d}{d \alpha_{i}} T\left(\alpha_{i}\right) \\
\partial_{\alpha_{i} \alpha_{j}}^{2} q(\alpha) & =k\left(x_{i}, x_{j}\right)+\delta_{i j} \frac{d^{2}}{d \alpha_{i}^{2}} T\left(\alpha_{i}\right) \\
\partial_{\alpha_{i} \alpha_{j}^{*}}^{2} q(\alpha) & =-k\left(x_{i}, x_{j}\right)
\end{aligned}
$$

and $\partial_{\alpha_{i}^{*} \alpha_{j}^{*}}^{2} q(\alpha), \partial_{\alpha_{i}^{*} \alpha_{j}}^{2} q(\alpha)$ analogously. Hence we are dealing with a matrix of type $M:=\left[{ }_{-}^{K}{ }_{K} D{ }_{K}^{K}+D^{\prime}\right]$ where $D, D^{\prime}$ are diagonal matrices. By applying an orthogonal transformation $M$ can be inverted essentially by inverting an $\ell \times \ell$ matrix instead of a $2 \ell \times 2 \ell$ system. This is exactly the additional advantage one can gain from implementing the optimization algorithm directly instead of using a general purpose optimizer. One can show that for practical implementations (Smola, Schölkopf and Müller 1998b) one can solve optimization problems using nearly arbitrary convex cost functions as efficiently as the special case of $\varepsilon$-insensitive loss functions.

Finally note that due to the fact that we are solving the primal and dual optimization problem simultaneously we are also 
computing parameters corresponding to the initial SV optimization problem. This observation is useful as it allows us to obtain the constant term $b$ directly, namely by setting $b=y$. (see Smola (1998) for details).

\section{Appendix B: Solving the subset selection problem}

\section{B.1. Subset optimization problem}

We will adapt the exposition of Joachims (1999) to the case of regression with convex cost functions. Without loss of generality we will assume $\varepsilon \neq 0$ and $\alpha \in[0, C]$ (the other situations can be treated as a special case). First we will extract a reduced optimization problem for the working set when all other variables are kept fixed. Denote $S_{w} \subset\{1, \ldots, \ell\}$ the working set and $S_{f}:=\{1, \ldots, \ell\} \backslash S_{w}$ the fixed set. Writing (43) as an optimization problem only in terms of $S_{w}$ yields

$\operatorname{maximize}\left\{\begin{aligned}- & \frac{1}{2} \sum_{i, j \in S_{w}}\left(\alpha_{i}-\alpha_{i}^{*}\right)\left(\alpha_{j}-\alpha_{j}^{*}\right)\left\langle x_{i}, x_{j}\right\rangle \\ & +\sum_{i \in S_{w}}\left(\alpha_{i}-\alpha_{i}^{*}\right)\left(y_{i}-\sum_{j \in S_{f}}\left(\alpha_{j}-\alpha_{j}^{*}\right)\left\langle x_{i}, x_{j}\right\rangle\right) \\ & +\sum_{i \in S_{w}}\left(-\varepsilon\left(\alpha_{i}+\alpha_{i}^{*}\right)+C\left(T\left(\alpha_{i}\right)+T\left(\alpha_{i}^{*}\right)\right)\right)\end{aligned}\right.$ subject to $\left\{\begin{array}{l}\sum_{i \in S_{w}}\left(\alpha_{i}-\alpha_{i}^{*}\right)=-\sum_{i \in S_{f}}\left(\alpha_{i}-\alpha_{i}^{*}\right) \\ \alpha_{i} \in[0, C]\end{array}\right.$

Hence we only have to update the linear term by the coupling with the fixed set $-\sum_{i \in S_{w}}\left(\alpha_{i}-\alpha_{i}^{*}\right) \sum_{j \in S_{f}}\left(\alpha_{j}-\alpha_{j}^{*}\right)\left\langle x_{i}, x_{j}\right\rangle$ and the equality constraint by $-\sum_{i \in S_{f}}\left(\alpha_{i}-\alpha_{i}^{*}\right)$. It is easy to see that maximizing (83) also decreases (43) by exactly the same amount. If we choose variables for which the KKT-conditions are not satisfied the overall objective function tends to decrease whilst still keeping all variables feasible. Finally it is bounded from below.

Even though this does not prove convergence (contrary to statement in Osuna, Freund and Girosi (1997)) this algorithm proves very useful in practice. It is one of the few methods (besides (Kaufman 1999, Platt 1999)) that can deal with problems whose quadratic part does not completely fit into memory. Still in practice one has to take special precautions to avoid stalling of convergence (recent results of Chang, Hsu and Lin (1999) indicate that under certain conditions a proof of convergence is possible). The crucial part is the one of $S_{w}$.

\section{B.2. A note on optimality}

For convenience the $K K T$ conditions are repeated in a slightly modified form. Denote $\varphi_{i}$ the error made by the current estimate at sample $x_{i}$, i.e.

$\varphi_{i}:=y_{i}-f\left(x_{i}\right)=y_{i}-\left[\sum_{j=1}^{m} k\left(x_{i}, x_{j}\right)\left(\alpha_{i}-\alpha_{i}^{*}\right)+b\right]$.

Rewriting the feasibility conditions (52) in terms of $\alpha$ yields

$$
\begin{array}{r}
2 \partial_{\alpha_{i}} T\left(\alpha_{i}\right)+\varepsilon-\varphi_{i}+s_{i}-z_{i}=0 \\
2 \partial_{\alpha_{i}^{*}} T\left(\alpha_{i}^{*}\right)+\varepsilon+\varphi_{i}+s_{i}^{*}-z_{i}^{*}=0
\end{array}
$$

for all $i \in\{1, \ldots, m\}$ with $z_{i}, z_{i}^{*}, s_{i}, s_{i}^{*} \geq 0$. A set of dual feasible variables $z, s$ is given by

$$
\begin{aligned}
z_{i} & =\max \left(2 \partial_{\alpha_{i}} T\left(\alpha_{i}\right)+\varepsilon-\varphi_{i}, 0\right) \\
s_{i} & =-\min \left(2 \partial_{\alpha_{i}} T\left(\alpha_{i}\right)+\varepsilon-\varphi_{i}, 0\right) \\
z_{i}^{*} & =\max \left(2 \partial_{\alpha_{i}^{*}} T\left(\alpha_{i}^{*}\right)+\varepsilon+\varphi_{i}, 0\right) \\
s_{i}^{*} & =-\min \left(2 \partial_{\alpha_{i}^{*}} T\left(\alpha_{i}^{*}\right)+\varepsilon+\varphi_{i}, 0\right)
\end{aligned}
$$

Consequently the KKT conditions (53) can be translated into

$$
\begin{array}{rll}
\alpha_{i} z_{i}=0 & \text { and } & \left(C-\alpha_{i}\right) s_{i}=0 \\
\alpha_{i}^{*} z_{i}^{*}=0 & \text { and } & \left(C-\alpha_{i}^{*}\right) s_{i}^{*}=0
\end{array}
$$

All variables $\alpha_{i}, \alpha_{i}^{*}$ violating some of the conditions of (87) may be selected for further optimization. In most cases, especially in the initial stage of the optimization algorithm, this set of patterns is much larger than any practical size of $S_{w}$. Unfortunately Osuna, Freund and Girosi (1997) contains little information on how to select $S_{w}$. The heuristics presented here are an adaptation of Joachims (1999) to regression. See also Lin (2001) for details on optimization for SVR.

\section{B.3. Selection rules}

Similarly to a merit function approach (El-Bakry et al. 1996) the idea is to select those variables that violate (85) and (87) most, thus contribute most to the feasibility gap. Hence one defines a score variable $\zeta_{i}$ by

$$
\begin{aligned}
\zeta_{i} & :=g_{i} z_{i}+s_{i} t_{i} \\
& =\alpha_{i} z_{i}+\alpha_{i}^{*} z_{i}^{*}+\left(C-\alpha_{i}\right) s_{i}+\left(C-\alpha_{i}^{*}\right) s_{i}^{*}
\end{aligned}
$$

By construction, $\sum_{i} \zeta_{i}$ is the size of the feasibility gap (cf. (56) for the case of $\varepsilon$-insensitive loss). By decreasing this gap, one approaches the the solution (upper bounded by the primal objective and lower bounded by the dual objective function). Hence, the selection rule is to choose those patterns for which $\zeta_{i}$ is 
largest. Some algorithms use

$$
\begin{aligned}
\zeta_{i}^{\prime}:= & \alpha_{i} \Theta\left(z_{i}\right)+\alpha_{i}^{*} \Theta\left(z_{i}^{*}\right) \\
& +\left(C-\alpha_{i}\right) \Theta\left(s_{i}\right)+\left(C-\alpha_{i}^{*}\right) \Theta\left(s_{i}\right) \\
\text { or } \quad \zeta_{i}^{\prime \prime}:= & \Theta\left(\alpha_{i}\right) z_{i}+\Theta\left(\alpha_{i}^{*}\right) z_{i}^{*} \\
& +\Theta\left(C-\alpha_{i}\right) s_{i}+\Theta\left(C-\alpha_{i}^{*}\right) s_{i} .
\end{aligned}
$$

One can see that $\zeta_{i}=0, \zeta_{i}^{\prime}=0$, and $\zeta_{i}^{\prime \prime}=0$ mutually imply each other. However, only $\zeta_{i}$ gives a measure for the contribution of the variable $i$ to the size of the feasibility gap.

Finally, note that heuristics like assigning sticky-flags (cf. Burges 1998) to variables at the boundaries, thus effectively solving smaller subproblems, or completely removing the corresponding patterns from the training set while accounting for their couplings (Joachims 1999) can significantly decrease the size of the problem one has to solve and thus result in a noticeable speedup. Also caching (Joachims 1999, Kowalczyk 2000) of already computed entries of the dot product matrix may have a significant impact on the performance.

\section{Appendix C: Solving the SMO equations}

\section{C.1. Pattern dependent regularization}

Consider the constrained optimization problem (83) for two indices, say $(i, j)$. Pattern dependent regularization means that $C_{i}$ may be different for every pattern (possibly even different for $\alpha_{i}$ and $\alpha_{i}^{*}$ ). Since at most two variables may become nonzero at the same time and moreover we are dealing with a constrained optimization problem we may express everything in terms of just one variable. From the summation constraint we obtain

$\left(\alpha_{i}-\alpha_{i}^{*}\right)+\left(\alpha_{j}-\alpha_{j}^{*}\right)=\left(\alpha_{i}^{\text {old }}-\alpha_{i}^{* \text { old }}\right)+\left(\alpha_{j}^{\text {old }}-\alpha_{j}^{* \text { old }}\right):=\gamma$

for regression. Exploiting $\alpha_{j}^{(*)} \in\left[0, C_{j}^{(*)}\right]$ yields $\alpha_{i}^{(*)} \in[L, H]$.

This is taking account of the fact that there may be only four different pairs of nonzero variables: $\left(\alpha_{i}, \alpha_{j}\right),\left(\alpha_{i}^{*}, \alpha_{j}\right),\left(\alpha_{i}, \alpha_{j}^{*}\right)$, and $\left(\alpha_{i}^{*}, \alpha_{j}^{*}\right)$. For convenience define an auxiliary variables $s$ such that $s=1$ in the first and the last case and $s=-1$ otherwise.

\begin{tabular}{llll}
\hline & & $\alpha_{j}$ & $\alpha_{j}^{*}$ \\
\hline$\alpha_{i}$ & $L$ & $\max \left(0, \gamma-C_{j}\right)$ & $\max (0, \gamma)$ \\
& $H$ & $\min \left(C_{i}, \gamma\right)$ & $\min \left(C_{i}, C_{j}^{*}+\gamma\right)$ \\
$\alpha_{i}^{*}$ & $L$ & $\max (0,-\gamma)$ & $\max \left(0,-\gamma-C_{j}^{*}\right)$ \\
& $H$ & $\min \left(C_{i}^{*},-\gamma+C_{j}\right)$ & $\min \left(C_{i}^{*},-\gamma\right)$ \\
\hline
\end{tabular}

\section{C.2. Analytic solution for regression}

Next one has to solve the optimization problem analytically. We make use of (84) and substitute the values of $\phi_{i}$ into the reduced optimization problem (83). In particular we use

$$
y_{i}-\sum_{j \notin S_{w}}\left(\alpha_{i}-\alpha_{i}^{*}\right) K_{i j}=\varphi_{i}+b+\sum_{j \in S_{w}}\left(\alpha_{i}^{\text {old }}-\alpha_{i}^{* \text { old }}\right) K_{i j} .
$$

Moreover with the auxiliary variables $\gamma=\alpha_{i}-\alpha_{i}^{*}+\alpha_{j}-\alpha_{j}^{*}$ and $\eta:=\left(K_{i i}+K_{j j}-2 K_{i j}\right)$ one obtains the following constrained optimization problem in $i$ (after eliminating $j$, ignoring terms independent of $\alpha_{j}, \alpha_{j}^{*}$ and noting that this only holds for $\alpha_{i} \alpha_{i}^{*}=$ $\left.\alpha_{j} \alpha_{j}^{*}=0\right)$ :

$$
\begin{aligned}
\operatorname{maximize}- & \frac{1}{2}\left(\alpha_{i}-\alpha_{i}^{*}\right)^{2} \eta-\varepsilon\left(\alpha_{i}+\alpha_{i}^{*}\right)(1-s) \\
& +\left(\alpha_{i}-\alpha_{i}^{*}\right)\left(\phi_{i}-\phi_{j}+\eta\left(\alpha_{i}^{\text {old }}-\alpha_{i}^{* \text { old }}\right)\right)
\end{aligned}
$$

subject to $\quad \alpha_{i}^{(*)} \in\left[L^{(*)}, H^{(*)}\right]$.

The unconstrained maximum of (92) with respect to $\alpha_{i}$ or $\alpha_{i}^{*}$ can be found below.

\begin{tabular}{lll}
\hline (I) & $\alpha_{i}, \alpha_{j}$ & $\alpha_{i}^{\text {old }}+\eta^{-1}\left(\varphi_{i}-\varphi_{j}\right)$ \\
(II) & $\alpha_{i}, \alpha_{j}^{*}$ & $\alpha_{i}^{\text {old }}+\eta^{-1}\left(\varphi_{i}-\varphi_{j}-2 \varepsilon\right)$ \\
(III) & $\alpha_{i}^{*}, \alpha_{j}$ & $\alpha_{i}^{* \text { old }}-\eta^{-1}\left(\varphi_{i}-\varphi_{j}+2 \varepsilon\right)$ \\
(IV) & $\alpha_{i}^{*}, \alpha_{j}^{*}$ & $\alpha_{i}^{* \text { old }}-\eta^{-1}\left(\varphi_{i}-\varphi_{j}\right)$ \\
\hline
\end{tabular}

The problem is that we do not know beforehand which of the four quadrants (I)-(IV) contains the solution. However, by considering the sign of $\gamma$ we can distinguish two cases: for $\gamma>0$ only (I)-(III) are possible, for $\gamma<0$ the coefficients satisfy one of the cases (II)-(IV). In case of $\gamma=0$ only (II) and (III) have to be considered. See also the diagram below.

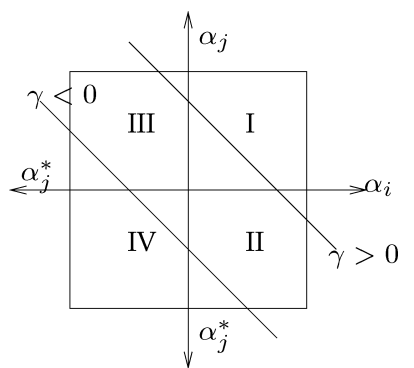

For $\gamma>0$ it is best to start with quadrant (I), test whether the unconstrained solution hits one of the boundaries $L, H$ and if so, probe the corresponding adjacent quadrant (II) or (III). $\gamma<0$ can be dealt with analogously.

Due to numerical instabilities, it may happen that $\eta<0$. In that case $\eta$ should be set to 0 and one has to solve (92) in a linear fashion directly. ${ }^{11}$ 


\section{C.3. Selection rule for regression}

Finally, one has to pick indices $(i, j)$ such that the objective function is maximized. Again, the reasoning of SMO (Platt 1999, Section 12.2.2) for classification will be mimicked. This means that a two loop approach is chosen to maximize the objective function. The outer loop iterates over all patterns violating the KKT conditions, first only over those with Lagrange multipliers neither on the upper nor lower boundary, and once all of them are satisfied, over all patterns violating the KKT conditions, to ensure self consistency on the complete dataset. ${ }^{12}$ This solves the problem of choosing $i$.

Now for $j$ : To make a large step towards the minimum, one looks for large steps in $\alpha_{i}$. As it is computationally expensive to compute $\eta$ for all possible pairs $(i, j)$ one chooses the heuristic to maximize the absolute value of the numerator in the expressions for $\alpha_{i}$ and $\alpha_{i}^{*}$, i.e. $\left|\varphi_{i}-\varphi_{j}\right|$ and $\left|\varphi_{i}-\varphi_{j} \pm 2 \varepsilon\right|$. The index $j$ corresponding to the maximum absolute value is chosen for this purpose.

If this heuristic happens to fail, in other words if little progress is made by this choice, all other indices $j$ are looked at (this is what is called "second choice hierarcy" in Platt (1999) in the following way:

1. All indices $j$ corresponding to non-bound examples are looked at, searching for an example to make progress on.

2. In the case that the first heuristic was unsuccessful, all other samples are analyzed until an example is found where progress can be made.

3. If both previous steps fail proceed to the next $i$.

For a more detailed discussion (see Platt 1999). Unlike interior point algorithms SMO does not automatically provide a value for $b$. However this can be chosen like in Section 1.4 by having a close look at the Lagrange multipliers $\alpha_{i}^{(*)}$ obtained.

\section{C.4. Stopping criteria}

By essentially minimizing a constrained primal optimization problem one cannot ensure that the dual objective function increases with every iteration step. ${ }^{13}$ Nevertheless one knows that the minimum value of the objective function lies in the interval [dual objective ${ }_{i}$, primal objective ${ }_{i}$ ] for all steps $i$, hence also in the interval $\left[\left(\max _{j \leq i}\right.\right.$ dual objective $\left._{j}\right)$, primal objective $\left.{ }_{i}\right]$. One uses the latter to determine the quality of the current solution.

The calculation of the primal objective function from the prediction errors is straightforward. One uses

$$
\sum_{i, j}\left(\alpha_{i}-\alpha_{i}^{*}\right)\left(\alpha_{j}-\alpha_{j}^{*}\right) k_{i j}=-\sum_{i}\left(\alpha_{i}-\alpha_{i}^{*}\right)\left(\varphi_{i}+y_{i}-b\right)
$$

i.e. the definition of $\varphi_{i}$ to avoid the matrix-vector multiplication with the dot product matrix.

\section{Acknowledgments}

This work has been supported in part by a grant of the DFG (Ja 379/71, Sm 62/1). The authors thank Peter Bartlett, Chris Burges, Stefan Harmeling, Olvi Mangasarian, Klaus-Robert Müller, Vladimir Vapnik, Jason Weston, Robert Williamson, and Andreas Ziehe for helpful discussions and comments.

\section{Notes}

1. Our use of the term 'regression' is somewhat lose in that it also includes cases of function estimation where one minimizes errors other than the mean square loss. This is done mainly for historical reasons (Vapnik, Golowich and Smola 1997).

2. A similar approach, however using linear instead of quadratic programming, was taken at the same time in the USA, mainly by Mangasarian $(1965,1968$, 1969).

3. See Smola (1998) for an overview over other ways of specifying flatness of such functions.

4. This is true as long as the dimensionality of $w$ is much higher than the number of observations. If this is not the case, specialized methods can offer considerable computational savings (Lee and Mangasarian 2001).

5. The table displays $C T(\alpha)$ instead of $T(\alpha)$ since the former can be plugged directly into the corresponding optimization equations.

6. The high price tag usually is the major deterrent for not using them. Moreover one has to bear in mind that in SV regression, one may speed up the solution considerably by exploiting the fact that the quadratic form has a special structure or that there may exist rank degeneracies in the kernel matrix itself.

7. For large and noisy problems (e.g. 100.000 patterns and more with a substantial fraction of nonbound Lagrange multipliers) it is impossible to solve the problem exactly: due to the size one has to use subset selection algorithms, hence joint optimization over the training set is impossible. However, unlike in Neural Networks, we can determine the closeness to the optimum. Note that this reasoning only holds for convex cost functions.

8. A similar technique was employed by Bradley and Mangasarian (1998) in the context of linear programming in order to deal with large datasets.

9. Due to length constraints we will not deal with the connection between Gaussian Processes and SVMs. See Williams (1998) for an excellent overview.

10. Strictly speaking, in Bayesian estimation one is not so much concerned about the maximizer $\hat{f}$ of $p(f \mid X)$ but rather about the posterior distribution of $f$.

11. Negative values of $\eta$ are theoretically impossible since $k$ satisfies Mercer's condition: $0 \leq\left\|\Phi\left(x_{i}\right)-\Phi\left(x_{j}\right)\right\|^{2}=K_{i i}+K_{j j}-2 K_{i j}=\eta$.

12. It is sometimes useful, especially when dealing with noisy data, to iterate over the complete KKT violating dataset already before complete self consistency on the subset has been achieved. Otherwise much computational resources are spent on making subsets self consistent that are not globally self consistent. This is the reason why in the pseudo code a global loop is initiated already when only less than $10 \%$ of the non bound variables changed.

13. It is still an open question how a subset selection optimization algorithm could be devised that decreases both primal and dual objective function at the same time. The problem is that this usually involves a number of dual variables of the order of the sample size, which makes this attempt unpractical.

\section{References}

Aizerman M.A., Braverman É.M., and Rozonoér L.I. 1964. Theoretical foundations of the potential function method in pattern recognition learning. Automation and Remote Control 25: 821-837.

Aronszajn N. 1950. Theory of reproducing kernels. Transactions of the American Mathematical Society 68: 337-404. 
Bazaraa M.S., Sherali H.D., and Shetty C.M. 1993. Nonlinear Programming: Theory and Algorithms, 2nd edition, Wiley.

Bellman R.E. 1961. Adaptive Control Processes. Princeton University Press, Princeton, NJ.

Bennett K. 1999. Combining support vector and mathematical programming methods for induction. In: Schölkopf B., Burges C.J.C., and Smola A.J., (Eds.), Advances in Kernel Methods-SV Learning, MIT Press, Cambridge, MA, pp. 307-326.

Bennett K.P. and Mangasarian O.L. 1992. Robust linear programming discrimination of two linearly inseparable sets. Optimization Methods and Software 1: 23-34.

Berg C., Christensen J.P.R., and Ressel P. 1984. Harmonic Analysis on Semigroups. Springer, New York.

Bertsekas D.P. 1995. Nonlinear Programming. Athena Scientific, Belmont, MA.

Bishop C.M. 1995. Neural Networks for Pattern Recognition. Clarendon Press, Oxford.

Blanz V., Schölkopf B., Bülthoff H., Burges C., Vapnik V., and Vetter T. 1996. Comparison of view-based object recognition algorithms using realistic 3D models. In: von der Malsburg C., von Seelen W., Vorbrüggen J.C., and Sendhoff B. (Eds.), Artificial Neural Networks ICANN'96, Berlin. Springer Lecture Notes in Computer Science, Vol. 1112, pp. 251-256.

Bochner S. 1959. Lectures on Fourier integral. Princeton Univ. Press, Princeton, New Jersey.

Boser B.E., Guyon I.M., and Vapnik V.N. 1992. A training algorithm for optimal margin classifiers. In: Haussler D. (Ed.), Proceedings of the Annual Conference on Computational Learning Theory. ACM Press, Pittsburgh, PA, pp. 144-152.

Bradley P.S., Fayyad U.M., and Mangasarian O.L. 1998. Data mining: Overview and optimization opportunities. Technical Report 98-01, University of Wisconsin, Computer Sciences Department, Madison, January. INFORMS Journal on Computing, to appear.

Bradley P.S. and Mangasarian O.L. 1998. Feature selection via concave minimization and support vector machines. In: Shavlik J. (Ed.), Proceedings of the International Conference on Machine Learning, Morgan Kaufmann Publishers, San Francisco, California, pp. 82-90. ftp://ftp.cs.wisc.edu/math-prog/tech-reports/9803.ps.Z.

Bunch J.R. and Kaufman L. 1977. Some stable methods for calculating inertia and solving symmetric linear systems. Mathematics of Computation 31: 163-179.

Bunch J.R. and Kaufman L. 1980. A computational method for the indefinite quadratic programming problem. Linear Algebra and Its Applications, pp. 341-370, December.

Bunch J.R., Kaufman L., and Parlett B. 1976. Decomposition of a symmetric matrix. Numerische Mathematik 27: 95-109.

Burges C.J.C. 1996. Simplified support vector decision rules. In L. Saitta (Ed.), Proceedings of the International Conference on Machine Learning, Morgan Kaufmann Publishers, San Mateo, CA, pp. 71-77.

Burges C.J.C. 1998. A tutorial on support vector machines for pattern recognition. Data Mining and Knowledge Discovery 2(2): 121167.

Burges C.J.C. 1999. Geometry and invariance in kernel based methods. In Schölkopf B., Burges C.J.C., and Smola A.J., (Eds.), Advances in Kernel Methods-Support Vector Learning, MIT Press, Cambridge, MA, pp. 89-116.
Burges C.J.C. and Schölkopf B. 1997. Improving the accuracy and speed of support vector learning machines. In Mozer M.C., Jordan M.I., and Petsche T., (Eds.), Advances in Neural Information Processing Systems 9, MIT Press, Cambridge, MA, pp. 375-381.

Chalimourda A., Schölkopf B., and Smola A.J. 2004. Experimentally optimal $v$ in support vector regression for different noise models and parameter settings. Neural Networks 17(1): 127-141.

Chang C.-C., Hsu C.-W., and Lin C.-J. 1999. The analysis of decomposition methods for support vector machines. In Proceeding of IJCAI99, SVM Workshop.

Chang C.C. and Lin C.J. 2001. Training v-support vector classifiers: Theory and algorithms. Neural Computation 13(9): 21192147.

Chen S., Donoho D., and Saunders M. 1999. Atomic decomposition by basis pursuit. Siam Journal of Scientific Computing 20(1): 33-61.

Cherkassky V. and Mulier F. 1998. Learning from Data. John Wiley and Sons, New York.

Cortes C. and Vapnik V. 1995. Support vector networks. Machine Learning 20: 273-297.

Cox D. and O'Sullivan F. 1990. Asymptotic analysis of penalized likelihood and related estimators. Annals of Statistics 18: 1676-1695.

CPLEX Optimization Inc. Using the CPLEX callable library. Manual, 1994.

Cristianini N. and Shawe-Taylor J. 2000. An Introduction to Support Vector Machines. Cambridge University Press, Cambridge, UK.

Cristianini N., Campbell C., and Shawe-Taylor J. 1998. Multiplicative updatings for support vector learning. NeuroCOLT Technical Report NC-TR-98-016, Royal Holloway College.

Dantzig G.B. 1962. Linear Programming and Extensions. Princeton Univ. Press, Princeton, NJ.

Devroye L., Györfi L., and Lugosi G. 1996. A Probabilistic Theory of Pattern Recognition. Number 31 in Applications of mathematics. Springer, New York.

Drucker H., Burges C.J.C., Kaufman L., Smola A., and Vapnik V. 1997. Support vector regression machines. In: Mozer M.C., Jordan M.I., and Petsche T. (Eds.), Advances in Neural Information Processing Systems 9, MIT Press, Cambridge, MA, pp. 155-161.

Efron B. 1982. The jacknife, the bootstrap, and other resampling plans. SIAM, Philadelphia.

Efron B. and Tibshirani R.J. 1994. An Introduction to the Bootstrap. Chapman and Hall, New York.

El-Bakry A., Tapia R., Tsuchiya R., and Zhang Y. 1996. On the formulation and theory of the Newton interior-point method for nonlinear programming. J. Optimization Theory and Applications 89: 507541.

Fletcher R. 1989. Practical Methods of Optimization. John Wiley and Sons, New York.

Girosi F. 1998. An equivalence between sparse approximation and support vector machines. Neural Computation 10(6): 1455-1480.

Girosi F., Jones M., and Poggio T. 1993. Priors, stabilizers and basis functions: From regularization to radial, tensor and additive splines. A.I. Memo No. 1430, Artificial Intelligence Laboratory, Massachusetts Institute of Technology.

Guyon I., Boser B., and Vapnik V. 1993. Automatic capacity tuning of very large VC-dimension classifiers. In: Hanson S.J., Cowan J.D., and Giles C.L. (Eds.), Advances in Neural Information Processing Systems 5. Morgan Kaufmann Publishers, pp. 147-155.

Härdle W. 1990. Applied nonparametric regression, volume 19 of Econometric Society Monographs. Cambridge University Press. 
Hastie T.J. and Tibshirani R.J. 1990. Generalized Additive Models, volume 43 of Monographs on Statistics and Applied Probability. Chapman and Hall, London.

Haykin S. 1998. Neural Networks: A Comprehensive Foundation. 2nd edition. Macmillan, New York.

Hearst M.A., Schölkopf B., Dumais S., Osuna E., and Platt J. 1998. Trends and controversies - support vector machines. IEEE Intelligent Systems 13: 18-28.

Herbrich R. 2002. Learning Kernel Classifiers: Theory and Algorithms. MIT Press.

Huber P.J. 1972. Robust statistics: A review. Annals of Statistics 43: 1041

Huber P.J. 1981. Robust Statistics. John Wiley and Sons, New York.

IBM Corporation. 1992. IBM optimization subroutine library guide and reference. IBM Systems Journal, 31, SC23-0519.

Jaakkola T.S. and Haussler D. 1999. Probabilistic kernel regression models. In: Proceedings of the 1999 Conference on AI and Statistics.

Joachims T. 1999. Making large-scale SVM learning practical. In: Schölkopf B., Burges C.J.C., and Smola A.J. (Eds.), Advances in Kernel Methods-Support Vector Learning, MIT Press, Cambridge, MA, pp. 169-184.

Karush W. 1939. Minima of functions of several variables with inequalities as side constraints. Master's thesis, Dept. of Mathematics, Univ. of Chicago.

Kaufman L. 1999. Solving the quadratic programming problem arising in support vector classification. In: Schölkopf B., Burges C.J.C., and Smola A.J. (Eds.), Advances in Kernel Methods-Support Vector Learning, MIT Press, Cambridge, MA, pp. 147-168

Keerthi S.S., Shevade S.K., Bhattacharyya C., and Murthy K.R.K. 1999. Improvements to Platt's SMO algorithm for SVM classifier design. Technical Report CD-99-14, Dept. of Mechanical and Production Engineering, Natl. Univ. Singapore, Singapore.

Keerthi S.S., Shevade S.K., Bhattacharyya C., and Murty K.R.K. 2001. Improvements to platt's SMO algorithm for SVM classifier design. Neural Computation 13: 637-649.

Kimeldorf G.S. and Wahba G. 1970. A correspondence between Bayesian estimation on stochastic processes and smoothing by splines. Annals of Mathematical Statistics 41: 495-502.

Kimeldorf G.S. and Wahba G. 1971. Some results on Tchebycheffian spline functions. J. Math. Anal. Applic. 33: 82-95.

Kowalczyk A. 2000. Maximal margin perceptron. In: Smola A.J., Bartlett P.L., Schölkopf B., and Schuurmans D. (Eds.), Advances in Large Margin Classifiers, MIT Press, Cambridge, MA, pp. 75113.

Kuhn H.W. and Tucker A.W. 1951. Nonlinear programming. In: Proc. 2nd Berkeley Symposium on Mathematical Statistics and Probabilistics, Berkeley. University of California Press, pp. 481-492.

Lee Y.J. and Mangasarian O.L. 2001. SSVM: A smooth support vector machine for classification. Computational optimization and Applications 20(1): 5-22.

Li M. and Vitányi P. 1993. An introduction to Kolmogorov Complexity and its applications. Texts and Monographs in Computer Science. Springer, New York.

Lin C.J. 2001. On the convergence of the decomposition method for support vector machines. IEEE Transactions on Neural Networks 12(6): 1288-1298.

Lustig I.J., Marsten R.E., and Shanno D.F. 1990. On implementing Mehrotra's predictor-corrector interior point method for linear pro- gramming. Princeton Technical Report SOR 90-03., Dept. of Civil Engineering and Operations Research, Princeton University.

Lustig I.J., Marsten R.E., and Shanno D.F. 1992. On implementing Mehrotra's predictor-corrector interior point method for linear programming. SIAM Journal on Optimization 2(3): 435449.

MacKay D.J.C. 1991. Bayesian Methods for Adaptive Models. PhD thesis, Computation and Neural Systems, California Institute of Technology, Pasadena, CA.

Mangasarian O.L. 1965. Linear and nonlinear separation of patterns by linear programming. Operations Research 13: 444-452.

Mangasarian O.L. 1968. Multi-surface method of pattern separation. IEEE Transactions on Information Theory IT-14: 801-807.

Mangasarian O.L. 1969. Nonlinear Programming. McGraw-Hill, New York.

Mattera D. and Haykin S. 1999. Support vector machines for dynamic reconstruction of a chaotic system. In: Schölkopf B., Burges C.J.C., and Smola A.J. (Eds.), Advances in Kernel MethodsSupport Vector Learning, MIT Press, Cambridge, MA, pp. 211242.

McCormick G.P. 1983. Nonlinear Programming: Theory, Algorithms, and Applications. John Wiley and Sons, New York.

Megiddo N. 1989. Progressin Mathematical Programming, chapter Pathways to the optimal set in linear programming, Springer, New York, NY, pp. 131-158.

Mehrotra S. and Sun J. 1992. On the implementation of a (primal-dual) interior point method. SIAM Journal on Optimization 2(4): 575601.

Mercer J. 1909. Functions of positive and negative type and their connection with the theory of integral equations. Philosophical Transactions of the Royal Society, London A 209: 415-446.

Micchelli C.A. 1986. Algebraic aspects of interpolation. Proceedings of Symposia in Applied Mathematics 36: 81-102.

Morozov V.A. 1984. Methods for Solving Incorrectly Posed Problems. Springer.

Müller K.-R., Smola A., Rätsch G., Schölkopf B., Kohlmorgen J., and Vapnik V. 1997. Predicting time series with support vector machines. In: Gerstner W., Germond A., Hasler M., and Nicoud J.-D. (Eds.), Artificial Neural Networks ICANN'97, Berlin. Springer Lecture Notes in Computer Science Vol. 1327 pp. 999-1004.

Murtagh B.A. and Saunders M.A. 1983. MINOS 5.1 user's guide. Technical Report SOL 83-20R, Stanford University, CA, USA, Revised 1987.

Neal R. 1996. Bayesian Learning in Neural Networks. Springer.

Nilsson N.J. 1965. Learning machines: Foundations of Trainable Pattern Classifying Systems. McGraw-Hill.

Nyquist. H. 1928. Certain topics in telegraph transmission theory. Trans. A.I.E.E., pp. 617-644.

Osuna E., Freund R., and Girosi F. 1997. An improved training algorithm for support vector machines. In Principe J., Gile L., Morgan N., and Wilson E. (Eds.), Neural Networks for Signal Processing VII-Proceedings of the 1997 IEEE Workshop, pp. 276-285, New York, IEEE.

Osuna E. and Girosi F. 1999. Reducing the run-time complexity in support vector regression. In: Schölkopf B., Burges C.J.C., and Smola A. J. (Eds.), Advances in Kernel Methods-Support Vector Learning, pp. 271-284, Cambridge, MA, MIT Press.

Ovari Z. 2000. Kernels, eigenvalues and support vector machines. Honours thesis, Australian National University, Canberra. 
Platt J. 1999. Fast training of support vector machines using sequential minimal optimization. In: Schölkopf B., Burges C.J.C., and Smola A.J. (Eds.) Advances in Kernel Methods-Support Vector Learning, pp. 185-208, Cambridge, MA, MIT Press.

Poggio T. 1975. On optimal nonlinear associative recall. Biological Cybernetics, 19: 201-209.

Rasmussen C. 1996. Evaluation of Gaussian Processes and Other Methods for Non-Linear Regression. PhD thesis, Department of Computer Science, University of Toronto,

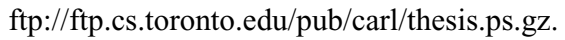

Rissanen J. 1978. Modeling by shortest data description. Automatica, 14: 465-471.

Saitoh S. 1988. Theory of Reproducing Kernels and its Applications. Longman Scientific \& Technical, Harlow, England.

Saunders C., Stitson M.O., Weston J., Bottou L., Schölkopf B., and Smola A. 1998. Support vector machine-reference manual. Technical Report CSD-TR-98-03, Department of Computer Science, Royal Holloway, University of London, Egham, UK. SVM available at http://svm.dcs.rhbnc.ac.uk/.

Schoenberg I. 1942. Positive definite functions on spheres. Duke Math. J., 9: 96-108.

Schölkopf B. 1997. Support Vector Learning. R. Oldenbourg Verlag, München. Doktorarbeit, TU Berlin. Download: http://www.kernel-machines.org.

Schölkopf B., Burges C., and Vapnik V. 1995. Extracting support data for a given task. In: Fayyad U.M. and Uthurusamy R. (Eds.), Proceedings, First International Conference on Knowledge Discovery \& Data Mining, Menlo Park, AAAI Press.

Schölkopf B., Burges C., and Vapnik V. 1996. Incorporating invariances in support vector learning machines. In: von der Malsburg C., von Seelen W., Vorbrüggen J. C., and Sendhoff B. (Eds.), Artificial Neural Networks ICANN'96, pp. 47-52, Berlin, Springer Lecture Notes in Computer Science, Vol. 1112.

Schölkopf B., Burges C.J.C., and Smola A.J. 1999a. (Eds.) Advances in Kernel Methods-Support Vector Learning. MIT Press, Cambridge, MA.

Schölkopf B., Herbrich R., Smola A.J., and Williamson R.C. 2001. A generalized representer theorem. Technical Report 2000-81, NeuroCOLT, 2000. To appear in Proceedings of the Annual Conference on Learning Theory, Springer (2001).

Schölkopf B., Mika S., Burges C., Knirsch P., Müller K.-R., Rätsch G., and Smola A. 1999b. Input space vs. feature space in kernel-based methods. IEEE Transactions on Neural Networks, 10(5): 10001017.

Schölkopf B., Platt J., Shawe-Taylor J., Smola A.J., and Williamson R.C. 2001. Estimating the support of a high-dimensional distribution. Neural Computation, 13(7): 1443-1471.

Schölkopf B., Simard P., Smola A., and Vapnik V. 1998a. Prior knowledge in support vector kernels. In: Jordan M.I., Kearns M.J., and Solla S.A. (Eds.) Advances in Neural Information Processing Systems 10, MIT Press. Cambridge, MA, pp. 640-646.

Schölkopf B., Smola A., and Müller K.-R. 1998b. Nonlinear component analysis as a kernel eigenvalue problem. Neural Computation, 10: 1299-1319.

Schölkopf B., Smola A., Williamson R.C., and Bartlett P.L. 2000. New support vector algorithms. Neural Computation, 12: 1207-1245.

Schölkopf B. and Smola A.J. 2002. Learning with Kernels. MIT Press.

Schölkopf B., Sung K., Burges C., Girosi F., Niyogi P., Poggio T., and Vapnik V. 1997. Comparing support vector machines with
Gaussian kernels to radial basis function classifiers. IEEE Transactions on Signal Processing, 45: 2758-2765.

Shannon C.E. 1948. A mathematical theory of communication. Bell System Technical Journal, 27: 379-423, 623-656.

Shawe-Taylor J., Bartlett P.L., Williamson R.C., and Anthony M. 1998. Structural risk minimization over data-dependent hierarchies. IEEE Transactions on Information Theory, 44(5): 19261940.

Smola A., Murata N., Schölkopf B., and Müller K.-R. 1998a. Asymptotically optimal choice of $\varepsilon$-loss for support vector machines. In: Niklasson L., Bodén M., and Ziemke T. (Eds.) Proceedings of the International Conference on Artificial Neural Networks, Perspectives in Neural Computing, pp. 105-110, Berlin, Springer.

Smola A., Schölkopf B., and Müller K.-R. 1998b. The connection between regularization operators and support vector kernels. Neural Networks, 11: 637-649.

Smola A., Schölkopf B., and Müller K.-R. 1998c. General cost functions for support vector regression. In: Downs T., Frean M., and Gallagher M. (Eds.) Proc. of the Ninth Australian Conf. on Neural Networks, pp. 79-83, Brisbane, Australia. University of Queensland.

Smola A., Schölkopf B., and Rätsch G. 1999. Linear programs for automatic accuracy control in regression. In: Ninth International Conference on Artificial Neural Networks, Conference Publications No. 470, pp. 575-580, London. IEE.

Smola. A.J. 1996. Regression estimation with support vector learning machines. Diplomarbeit, Technische Universität München.

Smola A.J. 1998. Learning with Kernels. PhD thesis, Technische Universität Berlin. GMD Research Series No. 25.

Smola A.J., Elisseeff A., Schölkopf B., and Williamson R.C. 2000. Entropy numbers for convex combinations and MLPs. In Smola A.J., Bartlett P.L., Schölkopf B., and Schuurmans D. (Eds.) Advances in Large Margin Classifiers, MIT Press, Cambridge, MA, pp. 369-387.

Smola A.J., Óvári Z.L., and Williamson R.C. 2001. Regularization with dot-product kernels. In: Leen T.K., Dietterich T.G., and Tresp V. (Eds.) Advances in Neural Information Processing Systems 13, MIT Press, pp. 308-314.

Smola A.J. and Schölkopf B. 1998a. On a kernel-based method for pattern recognition, regression, approximation and operator inversion. Algorithmica, 22: 211-231.

Smola A.J. and Schölkopf B. 1998b. A tutorial on support vector regression. NeuroCOLT Technical Report NC-TR-98-030, Royal Holloway College, University of London, UK.

Smola A.J. and Schölkopf B. 2000. Sparse greedy matrix approximation for machine learning. In: Langley P. (Ed.), Proceedings of the International Conference on Machine Learning, Morgan Kaufmann Publishers, San Francisco, pp. 911-918.

Stitson M., Gammerman A., Vapnik V., Vovk V., Watkins C., and Weston J. 1999. Support vector regression with ANOVA decomposition kernels. In: Schölkopf B., Burges C.J.C., and Smola A.J. (Eds.), Advances in Kernel Methods-Support Vector Learning, MIT Press Cambridge, MA, pp. 285-292.

Stone C.J. 1985. Additive regression and other nonparametric models. Annals of Statistics, 13: 689-705.

Stone M. 1974. Cross-validatory choice and assessment of statistical predictors (with discussion). Journal of the Royal Statistical Society, B36: 111-147. 
Street W.N. and Mangasarian O.L. 1995. Improved generalization via tolerant training. Technical Report MP-TR-95-11, University of Wisconsin, Madison.

Tikhonov A.N. and Arsenin V.Y. 1977. Solution of Ill-posed problems. V. H. Winston and Sons.

Tipping M.E. 2000. The relevance vector machine. In: Solla S.A., Leen T.K., and Müller K.-R. (Eds.), Advances in Neural Information Processing Systems 12, MIT Press, Cambridge, MA, pp. 652-658.

Vanderbei R.J. 1994. LOQO: An interior point code for quadratic programming. TR SOR-94-15, Statistics and Operations Research, Princeton Univ., NJ.

Vanderbei R.J. 1997. LOQO user's manual-version 3.10. Technical Report SOR-97-08, Princeton University, Statistics and Operations Research, Code available at http://www.princeton.edu/ rvdb/.

Vapnik V. 1995. The Nature of Statistical Learning Theory. Springer, New York.

Vapnik V. 1998. Statistical Learning Theory. John Wiley and Sons, New York.

Vapnik. V. 1999. Three remarks on the support vector method of function estimation. In: Schölkopf B., Burges C.J.C., and Smola A.J. (Eds.), Advances in Kernel Methods-Support Vector Learning, MIT Press, Cambridge, MA, pp. 25-42.

Vapnik V. and Chervonenkis A. 1964. A note on one class of perceptrons. Automation and Remote Control, 25.

Vapnik V. and Chervonenkis A. 1974. Theory of Pattern Recognition [in Russian]. Nauka, Moscow. (German Translation: Wapnik W. \& Tscherwonenkis A., Theorie der Zeichenerkennung, Akademie-Verlag, Berlin, 1979).

Vapnik V., Golowich S., and Smola A. 1997. Support vector method for function approximation, regression estimation, and signal processing. In: Mozer M.C., Jordan M.I., and Petsche T. (Eds.) Advances in Neural Information Processing Systems 9, MA, MIT Press, Cambridge. pp. 281-287.

Vapnik V. and Lerner A. 1963. Pattern recognition using generalized portrait method. Automation and Remote Control, 24: 774-780.
Vapnik V.N. 1982. Estimation of Dependences Based on Empirical Data. Springer, Berlin.

Vapnik V.N. and Chervonenkis A.Y. 1971. On the uniform convergence of relative frequencies of events to their probabilities. Theory of Probability and its Applications, 16(2): 264-281.

Wahba G. 1980. Spline bases, regularization, and generalized cross-validation for solving approximation problems with large quantities of noisy data. In: Ward J. and Cheney E. (Eds.), Proceedings of the International Conference on Approximation theory in honour of George Lorenz, Academic Press, Austin, TX, pp. 8-10.

Wahba G. 1990. Spline Models for Observational Data, volume 59 of CBMS-NSF Regional Conference Series in Applied Mathematics. SIAM, Philadelphia.

Wahba G. 1999. Support vector machines, reproducing kernel Hilbert spaces and the randomized GACV. In: Schölkopf B., Burges C.J.C., and Smola A.J. (Eds.), Advances in Kernel MethodsSupport Vector Learning, MIT Press, Cambridge, MA. pp. 69-88.

Weston J., Gammerman A., Stitson M., Vapnik V., Vovk V., and Watkins C. 1999. Support vector density estimation. In: Schölkopf B., Burges C.J.C., and Smola A.J. (Eds.) Advances in Kernel Methods-Support Vector Learning, MIT Press, Cambridge, MA. pp. 293-306.

Williams C.K.I. 1998. Prediction with Gaussian processes: From linear regression to linear prediction and beyond. In: Jordan M.I. (Ed.), Learning and Inference in Graphical Models, Kluwer Academic, pp. 599-621.

Williamson R.C., Smola A.J., and Schölkopf B. 1998. Generalization performance of regularization networks and support vector machines via entropy numbers of compact operators. Technical Report 19, NeuroCOLT, http://www.neurocolt.com. Published in IEEE Transactions on Information Theory, 47(6): 2516-2532 (2001).

Yuille A. and Grzywacz N. 1988. The motion coherence theory. In: Proceedings of the International Conference on Computer Vision, IEEE Computer Society Press, Washington, DC, pp. 344354. 